Noname manuscript No.

(will be inserted by the editor)

\title{
Improving Change Prediction Models with Code Smell-Related Information
}

\author{
Gemma Catolino - Fabio Palomba . \\ Francesca Arcelli Fontana • Andrea \\ De Lucia • Andy Zaidman • Filomena \\ Ferrucci
}

Received: date / Accepted: date

\begin{abstract}
Code smells represent sub-optimal implementation choices applied by developers when evolving software systems. The nagative impact of code smells has been widely investigated in the past: besides developers' productivity and ability to comprehend source code, researchers empirically showed that the presence of code smells heavily impacts the change-proneness of the affected classes. On the basis of these findings, in this paper we conjecture that code smell-related information can be effectively exploited to improve the performance of change prediction models, i.e., models having as goal that of indicating to developers which classes are more likely to change in the future, so that they may apply preventive maintenance actions. Specifically, we exploit the so-called intensity index - a previously defined metric that captures the severity of a code smell - and evaluate its contribution when added as additional feature in the context of three state of the art change prediction models based on product, process, and developer-based features. We also compare the performance achieved by the proposed model with the one of an alternative technique that considers the previously defined antipattern metrics, namely a set of indicators computed considering the history of code smells in files. Our results report that (i) the prediction performance of the intensity-including
\end{abstract}

Gemma Catolino, Andrea De Lucia, Filomena Ferrucci

University of Salerno, Italy

E-mail: gcatolino@unisa.it, adelucia@unisa.it, fferrucci@unisa.it

Fabio Palomba

University of Zurich, Switzerland

E-mail: palomba@ifi.uzh.ch

Francesca Arcelli Fontana

University of Milano-Bicocca, Italy

E-mail: arcelli@disco.unimib.it

Andy Zaidman

Delft University of Technology, The Netherlands

E-mail: a.e.zaidman@tudelft.nl 
models is statistically better than that of the baselines and (ii) the intensity is a more powerful metric with respect to the alternative smell-related ones. Nevertheless, we observed some complementarities between the set of changeprone and non-change-prone classes correctly classified by the models relying on intensity and antipattern metrics: for this reason, we devise and evaluate a smell-aware combined change prediction model including product, process, developer-based, and smell-related features. We show that this model has an F-Measure that is up to $20 \%$ higher than the existing state-of-the art models.

Keywords Change Prediction · Code Smells · Empirical Study

\section{Introduction}

During software evolution, change is unavoidable. Indeed, software systems are continuously modified in order to be adapted to changing needs, improved in performance or maintainability, or fixed from potential bugs [58. As a consequence, they become more complex, possibly eroding the original design with a subsequent reduction of their overall maintainability 88. In this context, predicting the source code components having a higher likelihood to change in the future represents an important activity to allow developers to plan preventive maintenance operations such as, e.g., refactoring [35] or peer-code reviews 7].

For this reason, the research community proposed several approaches in order to allow developers to control these changes [20, 27, 28, 38, 54, 159, 60, 94, 128. Such approaches are based on the usage of machine learning models exploiting several predictors that capture different characteristics of classes i.e., structural, process, and developer-related features.

Despite the good performance shown by those existing models, recent studies [52, 83. explored new factors contributing to the change-proneness of classes, finding that it is strongly influenced by the presence of the so-called bad code smells [35, i.e., sub-optimal design and/or implementation choices applied by practitioners when developing a software system. Specifically, such studies showed that smelly classes are significantly more likely to be the subject of changes than classes not affected by any design problem.

In this paper, we capture such findings and empirically investigate the extent to which smell-related information can be actually useful when considered in the context of the prediction of change-prone classes: our conjecture is that the addition of a measure of code smell severity can improve the performance of existing change prediction models, as it may help in the correct assessment of the change-proneness of classes. For severity, we mean a metric able to quantify how much a certain code smell instance is harmful for the design of a source code class. To test our conjecture, we (i) add the intensity index defined by Arcelli Fontana et al. 32 in three state of the art change prediction models based on structural [128, process [27, and developer-related metrics [20] and (ii) evaluate - on 43 releases of 14 large systems - how much such addition improves the prediction capabilities of the baseline models. We then compare 
the performance of the intensity-including change prediction models with the one achievable by exploiting alternative smell-related information such as the antipattern metrics defined by Taba et al. [110, which are able to capture historical information on code smell instances (e.g., the recurrence of a certain instance over time). The results show that the addition of the intensity index provides significant improvements in the performance of the baseline change prediction models, with an increase of $10 \%$ in terms of F-Measure on average. Furthermore, the intensity-including models work better than the models built adding the alternative antipattern metrics, although these two types of information are complementary, i.e., they correctly capture the change-proneness of different change-prone smelly classes.

Given such a complementarity, we then further explore the possibility to improve change prediction models by devising a smell-aware combined approach that mixes together the features of the experimented models, i.e., structural, process, developer-, and smell-related information, with the aim of boosting the change-proneness prediction abilities. As a result, we discovered that such a combined model is able to improve by up to $20 \%$ the performance of the baseline approaches.

To sum up, the contributions of this paper are the following:

1. A large-scale empirical assessment of the role of the intensity index 32 when predicting change-prone classes;

2. An empirical comparison between the capabilities of the intensity index and the antipattern metrics defined by Taba et al. [110] in the context of change prediction;

3. A novel smell-aware combined change prediction model, which has more effective performance than the state of the art;

4. A replication package that includes all the raw data and working data sets of our study [19].

Structure of the paper. Section 2 discusses the related literature on change prediction models and code smell. Section 3 describes the design of the case study aimed at evaluating the performance of the models, while Section 4 reports the results achieved. Section 5 discusses the threats to the validity of our empirical study. Finally, Section 6 concludes the paper and outlines directions for future work.

\section{Related Work}

Change-prone classes represent source code components that, for different reasons, tend to change more often than others. This phenomenon has been widely investigated by the research community [52, 68, 26, 14, 105, 83] with the aim of studying the factors contributing to the change-proneness of classes. Among all these studies, Khomh et al. [52] showed that the presence of sub-optimal implementations in Java classes, i.e., code smells, has a strong impact on the likelihood that such classes will be often modified by developers. The results 
were later confirmed by several studies in the field [22, 75, 83, 107, further highlighting the relevance of code smells for change-proneness. Our work is clearly based on these findings, and aims at providing additional evidence of how code smells can be adopted in the context of prediction models having the goal of identifying change-prone classes.

Keeping track of classes that are more prone to change can be relevant for two main reasons: on the one hand, to create awareness among developers about the fact that these classes tend to change frequently; on the other hand, to highlight the presence of classes that might require preventive maintenance actions (e.g., code review [7] or refactoring [35]) aimed at improving the quality of the source code. In this regard, previous researchers heavily investigated the feasibility of machine learning techniques for the identification of changeprone classes [20, 27, 28, 38, 54, 59, 60, 94, 128]. In the following, we discuss the advances achieved in the context of change prediction models. At the same time, as this paper reports on the role of code smell intensity, we also summarize the literature related to the detection and prioritization of code smells.

\subsection{Change Prediction Approaches}

The most relevant body of knowledge related to change prediction techniques is represented by the use of product and process metrics as independent variables able to characterize the change-proneness of software artifacts [2, 6, 17, 59, 60, 61, 118, 128. Specifically, Romano et al. 94 relied on code metrics for predicting change-prone the so-called fat interfaces (i.e., poorly-cohesive Java interfaces), while Eski et al. 28 proposed a model based on both CK and QMOOD metrics 11 to estimate change-prone classes and to determine parts of the source code that should be tested first and more deeply.

Conversely, Elish et al. 27] reported the potential usefulness of process metrics for change prediction. In particular, they defined a set of evolution metrics that describe the historical characteristics of software classes: for instance, they defined metrics like the birth date of a class or the total amount of changes applied in the past. As a result, their findings showed that a prediction model based on those evolution metrics can overcome the performance of structural-based techniques. These results were partially confirmed by Girba et al. 38, who defined a tool that suggests change-prone code elements by summarizing previous changes. In a small-scale empirical study involving two systems, they observed that previous changes can effectively predict future modifications.

More recently, Catolino et al. 20] empirically assessed the role of developerrelated factors in change prediction. To this aim, they studied the performance of three developer-based prediction models relying on (i) entropy of development process [45, (ii) number of developers working on a certain class 13, and (iii) structural and semantic scattering of changes 24, showing that they can be more accurate than models based on product or process metrics. Fur- 
thermore, they also defined a combined model which considers a mixture of metrics and that has shown to be up to $22 \%$ more accurate than the previously defined ones.

Our work builds upon the findings reported above. In particular, we study to what extent the addition of information related to the presence and severity of code smells can contribute to the performance of change prediction models based on product, process, and developer-based metrics.

Another consistent part of the state of the art concerns with the use of alternative methodologies to predict change-prone classes. For instance, the combination of (i) dependencies mined from UML diagrams and code metrics [43, 44, 96, 99, 100, and (ii) genetic and learning algorithms [62, 64, 89. have been proposed. Finally, some studies focused on the adoption of ensemble techniques for change prediction [18, 47, 55, 63. In particular, Malhotra and Khanna 63 proposed a search-based solution to the problem, adopting a Particle Swarm Optimization (PSO)-based classifier 47] for predicting the change-proneness of classes. The study was conducted on five Android application packages and the results encouraged the use of the adopted solution for developing change prediction models. Kumar et al. [55] studied the correlation between 62 software metrics and the likelihood of a class to change in the future. Afterwards, they built a change prediction model considering eight different machine learning algorithms and two ensemble techniques. The results showed that with the application of feature selection techniques, the change prediction models relying on ensemble classifiers can obtain better results. These results were partially contradicted by Catolino and Ferrucci [18, who empirically compared the performance of three ensemble techniques (i.e., , Boosting, Random Forest, and Bagging) with the one of standard machine learning classifiers (e.g., , Logistic Regression) on eight open source systems. The key results of the study showed how ensemble techniques in some cases perform better than standard machine learning approaches, however the differences among them is generally small.

\subsection{Code Smell Detection and Prioritization}

Fowler defined "bad code smells" (shortly, "code smells" or simply "smells") as "symptoms of the presence of poor design or implementation choices applied during the development of a software system" [35]. Starting from there, several researchers heavily investigated (i) how code smells evolve over time [84, 86, 91, 119, 120, 121, (ii) the way developers perceive them [79, 111, 126], and (iii) what is their impact on non-functional attributes of source code 11, 36, 50, 52, 77, 83, 104, 125. All these studies came up with a shared conclusion: code smells negatively impact program comprehension, maintainability of source code, and development costs. In the scope of this paper, the most relevant empirical studies are those reported by Khomh et al. [52] and Palomba et al. 83, who explicitly investigated the impact of code smells on software change proneness. Both the studies reported that classes affected by design 
flaws tend to change more frequently than classes not affected by any code smell. Moreover, refactoring practices notably help in keeping under control the change-proneness of classes. These studies are clearly those that motivate our work: indeed, following the findings on the influence of code smells, we believe that the addition of information coming from the analysis of the severity of code smells can positively improves the performance of change prediction models. As explained later in Section 5, we measure the intensity rather than the simple presence/absence of smells because a severity metric can provide us with a more fine-grained information on how much a design problem is "dangerous" for a certain source code class.

Starting from the findings on the negative impact of code smells on source code maintainability, the research community heavily focused on devising techniques able to automatically detect code smells. Most of these approaches rely on a two-step approach [51, 56, 65, 70, 72, 76, 117]: in the first one, a set of structural code metrics are computed and compared against predefined thresholds; in the second one, these metrics are combined using and/or operators in order to define detection rules. If the logical relationships expressed in such detection rules are violated, a code smell is identified. While these approaches already have good performance, Arcelli Fontana et al. 34 and Aniche et al. 3 proposed methods to further improve it by discarding false positive code smell instances or tailoring the thresholds of code metrics, respectively.

Besides structural analysis, the use of alternative sources of information for smell detection has been proposed. Ratiu et al. 93. and Palomba et al. 78, 80, showed how historical information can be exploited for detecting code smells. These approaches are particularly useful when dealing with design issues arising because of evolution problems (e.g., how a hierarchy evolves over time). On the other hand, Palomba et al. 82, 87 adopted Information Retrieval (IR) methods [8] to identify code smells characterized by promiscuous responsibilities (Blob classes).

Furthermore, Arcelli Fontana et al. [5, 31] and Kessentini et al. [16, 48, 49, 97. used machine learning and search-based algorithms to discover code smells, pointing out that a training set composed of one hundred instances is sufficient to reach very high values of accuracy. Nevertheless, Di Nucci et al. 25] recently showed that the performance of such techniques may vary depending on the exploited dataset.

Finally, Morales et al. [71] proposed a developer-based approach that leverages contextual information on the task a developer is currently working on to recommend what are the smells that can be removed on the portion of source code referring to the performed task.

In parallel with the definition of code smell detectors, several researchers faced the problem of prioritizing code smell instances based on their harmfulness for the overall maintainability of a software project. Vidal et al. [123] developed a semi-automated approach that recommends a ranking of code smells based on (i) past component modifications (e.g., number of changes during the system history), (ii) important modifiability scenarios, and (iii) relevance of the kind of smell assigned by developers. In a follow-up work, the 
same authors introduced a new criteria for prioritizing groups of code anomalies as indicators of architectural problems in evolving systems [122].

Lanza and Marinescu [56] proposed a metric-based rules approach in order to detect code smells, or identify code problems called disharmonies. The classes (or methods) that contain a high number of disharmonies are considered more critical. Marinescu [66] also presented the Flaw Impact Score, i.e., a measure of criticality of code smells that considers (i) negative influence of a code smell on coupling, cohesion, complexity, and encapsulation; (ii) granularity, namely the type of component (method or a class) that a smell affects; and (iii) severity, measured by one or more metrics analyzing the critical symptoms of the smell.

Murphy-Hill and Black [73] introduced an interactive visualization environment aimed at helping developers when assessing the harmfulness of code smell instances. The idea behind the tool is to visualize classes like petals, and an increase of code smell severity corresponded with an increased petal size. Other studies exploited developers' knowledge in order to assign a level of severity with the aim to suggest relevant refactoring solutions 69, while Zhao and Hayes [127] proposed a hierarchical approach to identify and prioritize refactoring operations based on predicted improvement to the maintainability of the software.

Besides the prioritization approaches mentioned above, more recently Arcelli Fontana and Zanoni [30] proposed the use of machine learning techniques to predict code smell severity, reporting promising results. The same authors also proposed JCODEODOR [32], a code smell detector that is able to assign a level of severity by computing the so-called intensity index, i.e., the extent to which a set of structural metrics computed on smelly classes exceed the predefined thresholds: the higher the distance between the actual and the threshold values the higher the severity of a code smell instance. As explained later in the paper (Section 3), in the context of our study we adopt JCODEODOR since it has been previously evaluated on the dataset we exploited, reporting a high accuracy. This was therefore the best option we had to conduct our work.

\section{Research Methodology}

In this section, we report the empirical study definition and design that we follow to test the contribution given by the addition of the code smell intensity index to existing change prediction models.

\subsection{Research Questions}

The goal of the empirical study was to evaluate the contribution of the intensity index in prediction models aimed at discovering change-prone classes, with the purpose of improving the allocation of resources in preventive maintenance task such as code inspection [7] or refactoring [35. The quality focus is on the 
prediction performance of models that include code smell-related information when compared to state of the art approaches, while the perspective is of researchers, who want to evaluate the effectiveness of using information about code smells when identifying change-prone components. More specifically, the empirical investigation aimed at answering the following research questions:

- $\mathbf{R Q}_{1}$. To what extent does the intensity index improve the performance of existing change prediction models?

- $\mathbf{R Q}_{2}$. How does the model including the intensity index as predictor compare to a model built using antipattern metrics?

- $\mathbf{R} \mathbf{Q}_{3}$. What is the gain provided by the intensity index to change prediction models when compared to other predictors?

- $\mathbf{R Q}_{4}$. What is the performance of a combined change prediction model that includes smell-related information?

As detailed in the next sections, the first research question $\left(\mathbf{R Q}_{1}\right)$ aimed at investigating the contribution given by the intensity index within change prediction models built using different types of predictors, i.e., product, process, and developer-related metrics. In $\mathbf{R Q}_{2}$ we empirically compared models relying on two different types of smell-related information, i.e., the intensity index [32] and the antipattern metrics proposed by Taba et al. [110]. $\mathbf{R Q}_{3}$ was concerned with a fine-grained analysis aimed at measuring the actual gain provided by the addition of the intensity metric within different change prediction models. Finally, $\mathbf{R Q}_{4}$ had the goal to assess the performance of a change prediction model built using a combination between smell-related information and other product, process, and developer-related features.

\subsection{Context Selection}

The context of the study was represented by a publicly available dataset coming from the Promise repository 67, which consisted of 43 releases of 14 open source software systems. Table 1 reports (i) the name of the considered projects, (ii) the number of releases for each of them, (iii) their size (min-max) in terms of minimum and maximum number of classes and KLOCs across the considered releases, (iv) the percentage (min-max) of change-prone classes (identified as explained later), and (iv) the percentage (min-max) of classes affected by design problems (detected as explained later). Their selection was driven by our willingness to analyze projects having different application domains and size in order to decrease threats to external validity [37, 106]. It is important to remark that the use of the Promise dataset required some additional effort in cleaning the data it contains: indeed, as reported by Shepperd et al. 101, such dataset may contain noise and/or erroneous entries that possibly negatively influence the results. To account for this aspect, before running our experiments we performed a data cleaning on the basis of the algorithm proposed by Shepperd et al. [101], which consists of 13 corrections 
Table 1: Characteristics of the Software Projects in Our Dataset

\begin{tabular}{lccccc}
\hline System & Releases & Classes & KLOCs & \% Change Cl. & \% Smelly Cl. \\
\hline Apache Ant & 5 & $83-813$ & $20-204$ & 24 & $11-16$ \\
Apache Camel & 4 & $315-571$ & $70-108$ & 25 & $9-14$ \\
Apache Forrest & 3 & $112-628$ & $18-193$ & 64 & $11-13$ \\
Apache Ivy & 1 & 349 & 58 & 65 & 12 \\
Apache Log4j & 3 & $205-281$ & $38-51$ & 26 & $15-19$ \\
Apache Lucene & 3 & $338-2,246$ & $103-466$ & 26 & $10-22$ \\
Apache Pbeans & 2 & $121-509$ & $13-55$ & 37 & $21-25$ \\
Apache POI & 4 & $129-278$ & $68-124$ & 22 & $15-19$ \\
Apache Synapse & 3 & $249-317$ & $117-136$ & 26 & $13-17$ \\
Apache Tomcat & 1 & 858 & 301 & 76 & 4 \\
Apache Velocity & 3 & $229-341$ & $57-73$ & 26 & $7-13$ \\
Apache Xalan & 4 & 909 & 428 & 25 & $5-9$ \\
Apache Xerces & 3 & $162-736$ & $62-201$ & 24 & $14-22$ \\
JEdit & 5 & $228-520$ & $39-166$ & 23 & \\
\hline
\end{tabular}

able to remove identical features, features with conflicting or missing values, etc. During this step, we removed 58 entries from the original dataset.

As for the code smells, our investigation copes with six types of design problems, namely:

- God Class (a.k.a., Blob): A poorly cohesive class that implements different responsibilities;

- Data Class: A class whose only purpose is holding data;

- Brain Method: A large method implementing more than one function, being therefore poorly cohesive;

- Shotgun Surgery: A class where every change triggers many little changes to several other classes;

- Dispersed Coupling: A class having too many relationships with other classes of the project;

- Message Chains: A method containing a long chain of method calls.

The choice of focusing on these specific smells was driven by two main aspects: (i) on the one hand, we took into account code smells characterizing different design problems (e.g., excessive coupling vs poorly cohesive classes/methods) and having different granularities; (ii) on the other hand, as explained in the next section, we could rely on a reliable tool to properly identify and compute their intensity in the classes of the exploited dataset.

\section{3 $\mathbf{R Q}_{1}$ - The contribution of the Intensity Index}

To answer our first research question, we needed to (i) identify code smells in the subject projects and compute their intensity, and (ii) select a set of existing change prediction models where to add the information on the intensity of code smells. Furthermore, we proceeded with the training and testing of the built change prediction models. The following subsections detail the process conducted to perform such steps. 
Table 2: Code Smell Detection Strategies (the complete names of the metrics are given in Table 3 and the explanation of the rules in Table 4 )

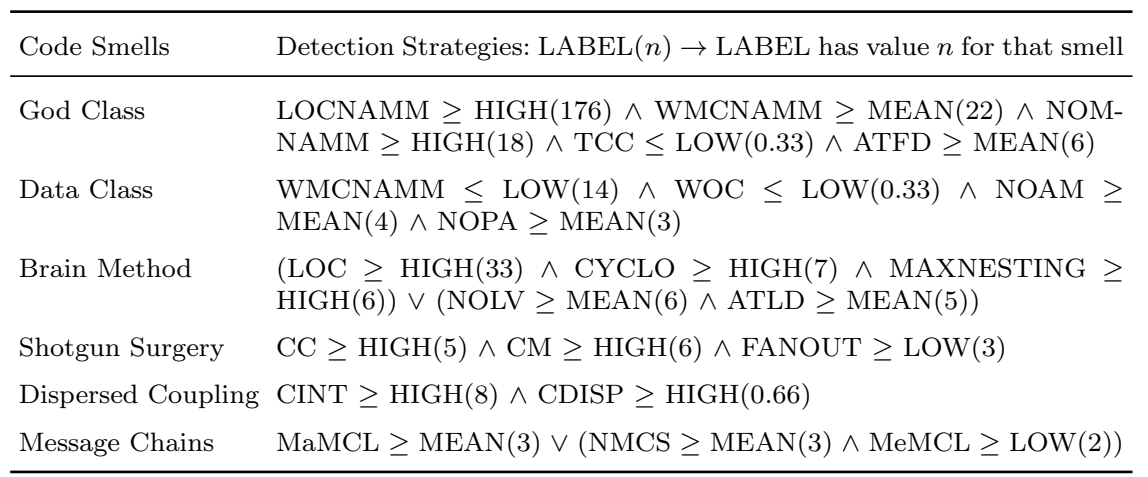

\subsubsection{Code Smell Intensity Computation}

To compute the severity of code smells in the context of our work we employed JCODEODor [32, a tool that is able to both identify code smells and assign to them a degree of severity by computing the so-called intensity index. Such an index is represented by a real number contained in the range $[1,10]$. Before reporting the detailed steps adopted by JCODEODOR to compute the intensity index, it is worth remarking that our choice to use this tool was driven by two observations. In the first place, JCODEODOR works with all the code smells considered in our work: in literature it is the prioritization approach that deal with the highest number of code smells ( 6 vs the 5 treated by Vidal et al. [123]). Moreover, it is fully automated, meaning that it does not require any human intervention while computing the intensity of code smells. Finally, it is highly accurate: in a previous work by Palomba et al. 85 the tool was empirically assessed on the same dataset adopted in our context, showing an F-Measure of $80 \%$. For these reasons, we believe that JCODEODOR was the best option we had to conduct our study.

From a technical point of view, given the set of classes composing a certain software system the tool performs two basic steps to compute the intensity of code smells:

1. Detection Phase. Given a software system as input, the tool starts by detecting code smells relying on the detection strategies reported in Table 2 . Basically, each strategy is represented by a logical composition of predicates, and each predicate is based on an operator that compares a metric with a threshold [56, 81]. Such detection strategies are similar to those defined by Lanza and Marinescu [56], who used the set of code metrics described in Table 3 to identify the six code smell types in our study. To ease the comprehension of the detection approach, Table 4 describes the rationale behind the use of each predicate of the detection strategies. 
Table 3: Metrics used for Code Smells Detection

\begin{tabular}{|c|c|c|}
\hline Short Name & Long Name & Definition \\
\hline ATFD & $\begin{array}{l}\text { Access To Foreign } \\
\text { Data }\end{array}$ & $\begin{array}{l}\text { The number of attributes from unrelated classes be- } \\
\text { longing to the system, accessed directly or by invoking } \\
\text { accessor methods. }\end{array}$ \\
\hline ATLD & $\begin{array}{lll}\text { Access } & \text { To } & \text { Local } \\
\text { Data } & & \end{array}$ & $\begin{array}{l}\text { The number of attributes declared by the current } \\
\text { classes accessed by the measured method directly or } \\
\text { by invoking accessor methods. }\end{array}$ \\
\hline $\mathrm{CC}$ & Changing Classes & $\begin{array}{l}\text { The number of classes in which the methods that call } \\
\text { the measured method are defined in. }\end{array}$ \\
\hline CDISP & $\begin{array}{l}\text { Coupling Disper- } \\
\text { sion }\end{array}$ & $\begin{array}{l}\text { The number of classes in which the operations called } \\
\text { from the measured operation are defined, divided by } \\
\text { CINT. }\end{array}$ \\
\hline CINT & Coupling Intensity & $\begin{array}{l}\text { The number of distinct operations called by the mea- } \\
\text { sured operation. }\end{array}$ \\
\hline $\mathrm{CM}$ & Changing Methods & $\begin{array}{l}\text { The number of distinct methods that call the mea- } \\
\text { sured method. }\end{array}$ \\
\hline CYCLO & $\begin{array}{l}\text { McCabe Cyclo- } \\
\text { matic Complexity }\end{array}$ & $\begin{array}{l}\text { The maximum number of linearly independent paths } \\
\text { in a method. A path is linear if there is no branch in } \\
\text { the execution flow of the corresponding code. }\end{array}$ \\
\hline FANOUT & & Number of called classes. \\
\hline $\mathrm{LOC}$ & Lines Of Code & $\begin{array}{l}\text { The number of lines of code of an operation or of a } \\
\text { class, including blank lines and comments. }\end{array}$ \\
\hline LOCNAMM & $\begin{array}{lr}\text { Lines of } & \text { Code } \\
\text { Without } & \text { Acces- } \\
\text { sor or Mutator } \\
\text { Methods }\end{array}$ & $\begin{array}{l}\text { The number of lines of code of a class, including blank } \\
\text { lines and comments and excluding accessor and muta- } \\
\text { tor methods and corresponding comments. }\end{array}$ \\
\hline MaMCL & $\begin{array}{l}\text { Maximum Message } \\
\text { Chain Length }\end{array}$ & The maximum length of chained calls in a method. \\
\hline MAXNESTING & $\begin{array}{l}\text { Maximum Nesting } \\
\text { Level }\end{array}$ & $\begin{array}{l}\text { The maximum nesting level of control structures } \\
\text { within an operation. }\end{array}$ \\
\hline MeMCL & $\begin{array}{l}\text { Mean Message } \\
\text { Chain Length }\end{array}$ & The average length of chained calls in a method. \\
\hline NMCS & $\begin{array}{l}\text { Number of Mes- } \\
\text { sage Chain State- } \\
\text { ments }\end{array}$ & The number of different chained calls in a method. \\
\hline NOAM & $\begin{array}{l}\text { Number Of Acces- } \\
\text { sor Methods }\end{array}$ & $\begin{array}{l}\text { The number of accessor (getter and setter) methods of } \\
\text { a class. }\end{array}$ \\
\hline NOLV & $\begin{array}{l}\text { Number Of Local } \\
\text { Variables }\end{array}$ & $\begin{array}{l}\text { Number of local variables declared in a method. The } \\
\text { method's parameters are considered local variables. }\end{array}$ \\
\hline NOMNAMM & $\begin{array}{l}\text { Number of Not Ac- } \\
\text { cessor or Mutator } \\
\text { Methods }\end{array}$ & $\begin{array}{l}\text { The number of methods defined locally in a class, } \\
\text { counting public as well as private methods, exclud-ing } \\
\text { accessor or mutator methods. }\end{array}$ \\
\hline${ }^{*}$ NOMNAMM & $\begin{array}{l}\text { Number of Not Ac- } \\
\text { cessor or Mutator } \\
\text { Methods }\end{array}$ & $\begin{array}{l}\text { The number of methods defined locally in a class, } \\
\text { counting public as well as private methods, excluding } \\
\text { accessor or mutator methods. }\end{array}$ \\
\hline NOPA & $\begin{array}{l}\text { Number Of Public } \\
\text { Attributes }\end{array}$ & The number of public attributes of a class. \\
\hline TCC & $\begin{array}{l}\text { Tight Class Cohe- } \\
\text { sion }\end{array}$ & $\begin{array}{l}\text { The normalized ratio between the number of meth- } \\
\text { ods directly connected with other methods through } \\
\text { an instance variable and the total number of possi- } \\
\text { ble connections between methods. A direct connection } \\
\text { between two methods exists if both access the same in- } \\
\text { stance variable directly or indirectly through a method } \\
\text { call. TCC takes its value in the range }[0,1] \text {. }\end{array}$ \\
\hline WMCNAMM & $\begin{array}{l}\text { Weighted Methods } \\
\text { Count of Not Ac- } \\
\text { cessor or Mutator } \\
\text { Methods }\end{array}$ & $\begin{array}{l}\text { The sum of complexity of the methods that are defined } \\
\text { in the class, and are not accessor or mutator meth- } \\
\text { ods. We compute the complexity with the Cyclomatic } \\
\text { Complexity metric (CYCLO). }\end{array}$ \\
\hline WOC & Weight Of Class & $\begin{array}{l}\text { The number of "functional" (i.e., non-abstract, non- } \\
\text { accessor, non-mutator) public methods divided by the } \\
\text { total number of public members. }\end{array}$ \\
\hline
\end{tabular}


Table 4: Code Smell Detection Rationale and Details

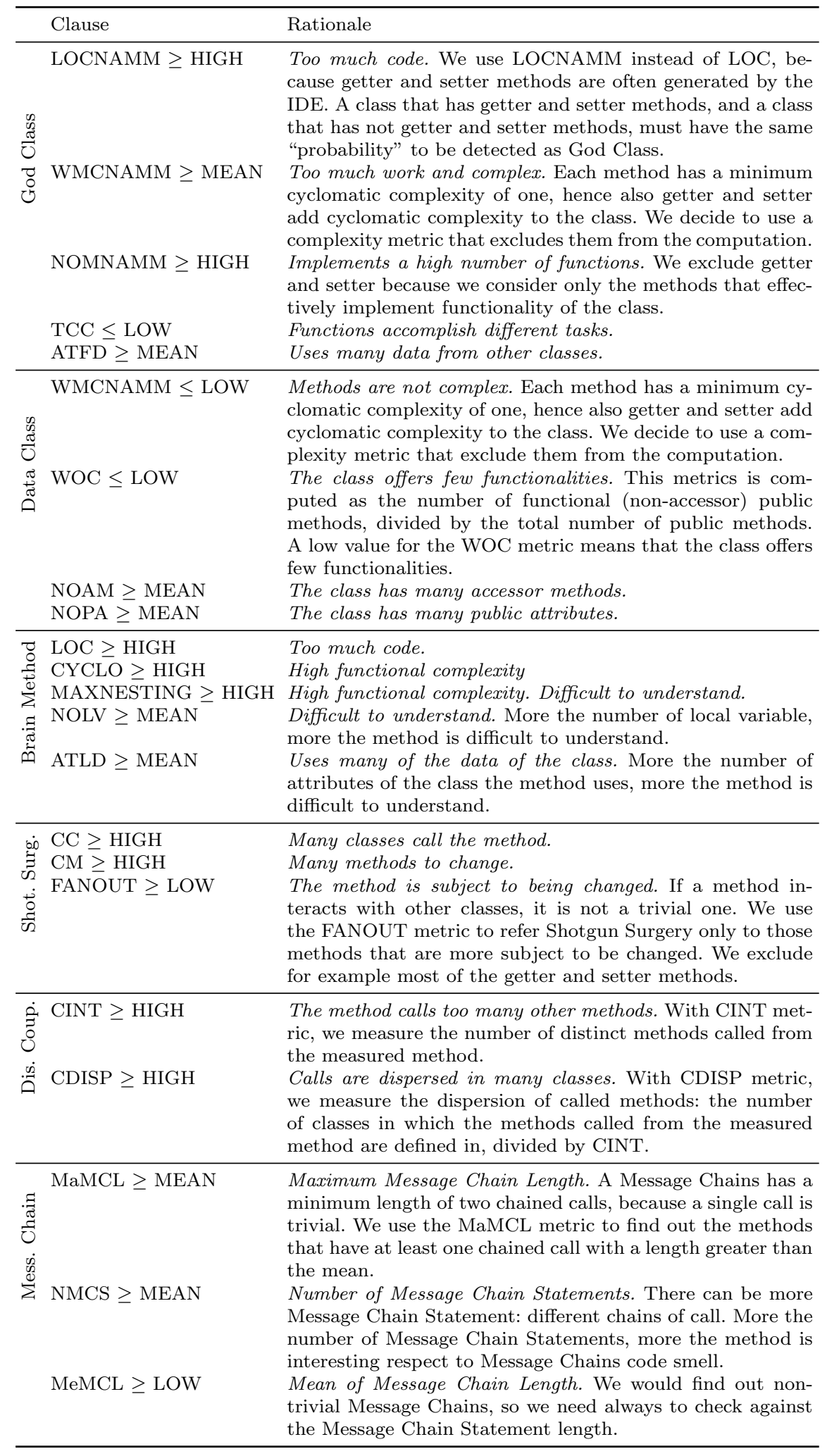


On the basis of these detection rules, a class/method of a project is marked as smelly if one of the logical propositions shown in Table 2 is true, i.e., if the actual metrics computed on the class/method exceed the threshold values defined in the detection strategy. It is worth pointing out that the thresholds used by JCODEODOR were empirically calibrated on 74 systems belonging to the QUALITAS CORPUS dataset [115] and are derived from the statistical distribution of the metrics contained in the dataset [32, 33.

2. Intensity Computation. If a class/method is identified by the tool as smelly, the actual value of a given metric used for the detection will exceed the threshold value, and it will correspond to a percentile value on the metric distribution placed between the threshold and the maximum observed value of the metric in the system under analysis. The placement of the actual metric value in that range represents the "exceeding amount" of a metric with respect to the defined threshold. Such "exceeding amounts" are then normalized in the range $[1,10]$ using a min-max normalization process [116: specifically, this is a feature scaling technique where the values of a numeric range are reduced to a scale between 1 and 10 . To compute $z$, i.e., the normalized value, the following formula is applied:

$$
z=\left[\frac{x-\min (x)}{\max (x)-\min (x)}\right] \cdot 10
$$

where min and $\max$ are the minimum and maximum values observed in the distribution. This step allows to have the "exceeding amount" of each metric in the same scale. To have a unique value representing the intensity of the code smell affecting the class, the mean of the normalized "exceeding amounts" is computed.

\subsubsection{Selection of Basic Prediction Models}

Our conjecture was concerned with the gain given by the addition of information on the intensity of code smells within existing change prediction models. To test such a conjecture, we needed to identify the state of the art techniques where to add the intensity index: we selected three models based on product, process, and developer-related metrics that were shown to be accurate in the context of change prediction [20, 24, 27, 128.

Product Metrics-based Model. The first basic baseline is represented by the change prediction model devised by Zhou et al. 128. It is composed of a set of metrics computed on the basis of the structural properties of source code: these are cohesion (i.e., the Lack of Cohesion of Method - LCOM), coupling (i.e., the Coupling Between Objects - CBO - and the Response for a Class - RFC), and inheritance metrics (i.e., the Depth of Inheritance Tree - DIT). To actually compute these metrics, we relied on a publicly available tool originally developed by Spinellis [108. In the following, we refer to this model as SM, i.e., Structural Model. 
Table 5: Independent variables considered by Elish et al.

\begin{tabular}{ll}
\hline Acronym & Metric \\
\hline BOC & Birth of a Class \\
FCH & First Time Changes Introduced to a Class \\
LCH & Frequency of Changes \\
WCD & Last Time Changes Introduced to a Class \\
WFR & Weighted Change Density \\
TACH & Weighted Frequency of Changes \\
ATAF & Total Amount of Changes \\
& Aggregated Change Size Normalized by \\
CHD & Frequency of Change \\
LCA & Change Density \\
LCD & Last Change Amount \\
CSB & Last Change Density \\
CSBS & Changes since the Birth \\
ACDF & Changes since the Birth Normalized by \\
& Size \\
CHO & Aggregated Change Density Normalized \\
& by Frequency of Change \\
\hline
\end{tabular}

Process Metrics-based Model. In their study, Elish et al. 27 reported that process metrics can be exploited as better predictors of change-proneness with respect to structural metrics. For this reason, our second baseline was the Evolution Model (EM) proposed by Elish et al. [27. More specifically, this model relies on the metrics shown in Table 5 , which capture different aspects of the evolution of classes, e.g., the weighted frequency of changes or the first time changes introduced. To compute these metrics, we adopted the tool that was previously developed by Catolino et al. 20]. In the following, we refer to this model as PM, i.e., Process Model.

Developer-Related Model. In our previous work [20], we demonstrated how developer-related factors can be exploited within change prediction models since they provide orthogonal information with respect to product and process metrics that takes into account how developers perform modifications and how complex the development process is. Among all the available developerbased models developed in literature [13, 24, 45, in this paper we relied on the Developer Changes Based Model (DCBM) devised by Di Nucci et al. 24, as it was shown to be the most effective one in the context of change prediction. Such a model uses as predictors the so-called structural and semantic scattering of the developers that worked on a code component in a given time period $\alpha$. Specifically, for each class $c$, the two metrics are computed as follows:

$$
\begin{aligned}
\text { StrScatPred }_{c, \alpha} & =\sum_{d \in \text { developer }_{c, \alpha}} \text { StrScat }_{d, \alpha} \\
\text { SemScatPred }_{c, \alpha} & =\sum_{d \in \text { developer }_{c, \alpha}} \text { SemScat }_{d, \alpha}
\end{aligned}
$$


where developer $s_{c, \alpha}$ represents the set of developers that worked on the class $c$ during a certain period $\alpha$, and the functions $S_{t r S c a t}{ }_{d, \alpha}$ and $\operatorname{SemScat}_{d, \alpha}$ return the structural and semantic scattering, respectively, of a developer $d$ in the time window $\alpha$. Given the set $C H_{d, \alpha}$ of classes changed by a developer $d$ during a certain period $\alpha$, the formula of structural scattering of a developer is:

$$
\operatorname{StrScat}_{d, \alpha}=\left|C H_{d, \alpha}\right| \times \underset{\forall c_{i}, c_{j} \in C H_{d, \alpha}}{\operatorname{average}}\left[\operatorname{dist}\left(c_{i}, c_{j}\right)\right]
$$

where dist is the distance in number of packages from class $c_{i}$ to class $c_{j}$. The structural scattering is computed by applying the shortest path algorithm on the graph representing the systems package structure. Regarding the semantic scattering of a developer, it is based on the textual similarity of the classes changed by a developer in a certain period $\alpha$ and it is computed as:

$$
\text { SemScat }_{d, \alpha}=\left|C H_{d, \alpha}\right| \times \frac{1}{\begin{array}{c}
\text { average } \\
\forall c_{i}, c_{j} \in C H_{d, \alpha}
\end{array}}\left[\operatorname{sim}\left(c_{i}, c_{j}\right)\right]
$$

where the sim function returns the textual similarity between the classes $c_{i}$ and $c_{j}$ according to the measurement performed using the Vector Space Model (VSM) 9. The metric ranges between zero (no textual similarity) and one (the textual content of the two classes is identical). In our study, we set the parameter $\alpha$ of the approach as the time window between two releases $R-1$ and $R$, as done in previous work 85$]$.

It is important to note that all the baseline models might be affected by multi-collinearity 74, which occurs when two or more independent variables are highly correlated and can be predicted one from the other, thus possibly leading to a decrease of the prediction capabilities of the resulting model $[102$, 113. For this reason, we decided to use the vif (variance inflation factors) function 74 implemented in $\mathrm{R}^{1}$ to discard non-relevant variables. Vif is based on the square of the multiple correlation coefficient resulting from regressing a predictor variable against all other predictor variables. If a variable has a strong linear relationship with at least one other variable, the correlation coefficient would be close to 1 , and VIF for that variable would be large. A VIF greater than 10 is a signal that the model has a collinearity problem.

\subsubsection{Dependent Variable}

Our dependent variable is represented by the actual change-proneness of the classes in our dataset. To compute it, we followed the guidelines provided by Romano et al. 94, who considered a class as change-prone if, in a given time period $T W$, it underwent a number of changes higher than the median of the distribution of the number of changes experienced by all the classes of the system. In particular, for each pair of commits $\left(c_{i}, c i+1\right)$ of $T W$ we run

1 http://cran.r-project.org/web/packages/car/index.html 
Table 6: Changes extracted by CHAngeDistiller while computing the change-proneness. ' $\checkmark$ ' symbols indicate the types we considered in our study.

\begin{tabular}{lc} 
ChangeDistiller & Our Study \\
Statement-level changes & \\
Statement Ordering Change & $\checkmark$ \\
Statement Parent Change & $\checkmark$ \\
Statement Insert & $\checkmark$ \\
Statement Delete & $\checkmark$ \\
Statement Update & $\checkmark$ \\
Class-body changes & $\checkmark$ \\
Insert attribute & $\checkmark$ \\
Delete attribute & \\
Declaration-part changes & $\checkmark$ \\
Access modifier update & $\checkmark$ \\
Final modifier update & \\
Declaration-part changes & $\checkmark$ \\
Increasing accessibility change & $\checkmark$ \\
Decreasing accessibility change & $\checkmark$ \\
Final Modified Insert & $\checkmark$ \\
Final Modified Delete & \\
Attribute declaration changes & $\checkmark$ \\
Attribute type change & $\checkmark$ \\
Attribute renaming change & \\
\hline Method declaration changes & $\checkmark$ \\
Return type insert & $\checkmark$ \\
Return type delete & $\checkmark$ \\
Return type update & $\checkmark$ \\
Method renaming & $\checkmark$ \\
Parameter insert & $\checkmark$ \\
Parameter delete & $\checkmark$ \\
Parameter ordering change & $\checkmark$ \\
Parameter renaming & $\checkmark$ \\
Class declaration changes & \\
Class renaming & $\checkmark$ \\
Parent class insert & \\
Parent class delete & \\
Parent class update & \\
\hline & \\
\hline
\end{tabular}

ChangeDistiller [29, a tree differencing algorithm able to extract the finegrained code changes between $c_{i}$ and $c i+1$. Table 6 reports the entire list of change types identified by the tool. As it is possible to observe, we considered all of them while computing the number of changes. It is worth mentioning that the tool ignores white space-related differences and documentation-related updates: in this way, it only considers the changes actually applied on the source code. More importantly, CHANGEDistiller is able to identify rename refactoring operations: this means that we could handle cases where a class was modified during the change history, thus not biasing the correct counting of the number of changes. The dataset with the oracle is available on the online appendix [19.

\subsubsection{Experimented Machine Learning Models}

Once we had defined dependent and independent variables of interest, we could finally build machine learning models. As we were interested in understanding and measuring the contribution given by the intensity within the three base- 
lines, we built two types of prediction models for each baseline: the first type of model does not include the intensity index as predictor, thus relying on the original features only; the second type of model that includes the intensity index as an additional predictor. Using this procedure, we experimented with 6 different models, and we could control the actual amount of improvement given by the intensity index with respect to the baselines (if any). It is worth remarking that, for non-smelly classes, the intensity value is set to 0 .

\subsubsection{Classifier Selection}

The final step of our prediction model construction methodology was concerned with the selection of the best machine learning classifier able to distinguish change-prone and non-change-prone classes. The current literature proposed several alternatives (e.g., Romano and Pinzger 94 adopted Support Vector Machines [15, while Tsantalis et al. 118, relied on Logistic Regression [57]), and thus there is not a bullet-proof solution that ensures the best overall performance. For this reason, in our work we experimented with different classifiers, i.e., ADTree [124, Decision Table Majority [53, Logistic Regression [21, Multilayer Perceptron [95], Naive Bayes [46], and Simple Logistic Regression [90]. To select the right classifier to use in our situation, we empirically compared the results achieved when applying each classifier on each experimented model on the software systems in our study. Overall, the best results were obtained using the Simple Logistic Regression. In the remaining of the paper, we only report the results obtained when using this classifier, while a complete report of the performance of other classifiers is available in our online appendix [19].

\subsubsection{Validation Strategy}

As for validation strategy we adopted 10-Fold Cross Validation 109. This methodology randomly partitions the data into 10 folds of equal size, applying a stratified sampling. A single fold is used as test set, while the remaining ones are used as training set. The process was repeated 10 times, using each time a different fold as test set. Then, the model performances were reported using the mean achieved over the ten runs. It is important to note that we repeated the 10-fold validation 100 times (each time with a different seed) to cope with the randomness arising from using different data splits [42].

\subsubsection{Evaluation Metrics}

To measure and compare the performance of the models, we started computing two well-known metrics such as precision and recall [10, which are defined as follow:

$$
\text { precision }=\frac{T P}{T P+F P} \quad \text { recall }=\frac{T P}{T P+T N}
$$


where $T P$ is the number of true positives, $T N$ the number of true negatives, and $F P$ the number of false positives. In the second place, to have a unique value representing the goodness of the model, we computed the F-Measure, i.e., the harmonic mean of precision and recall:

$$
F-\text { Measure }=2 * \frac{\text { precision } * \text { recall }}{\text { precision }+ \text { recall }}
$$

Moreover, we considered another indicator: the Area Under the ROC Curve (AUC-ROC) metric. This measure quantifies the overall ability of a change prediction model to discriminate between change-prone and non-change-prone classes: the closer the AUC-ROC to 1 the higher the ability of the classifier, while the closer the AUC-ROC to 0.5 the lower its accuracy. In other words, this metric can quantity how rubust the model is when discriminating the two binary classes.

In addition, we compared the performance achieved by the experimented prediction models from a statistical point of view. As we performed comparisons over multiple datasets, we employed the Scott-Knott Effect Size Difference (ESD) test [112, 114, considering the AUC-ROC that the different models obtained over the considered systems. This test represents an effectsize aware variant of Scott-Knott test [98]: differently from the original one, it (i) hierarchically clusters the set of treatment means into statistically distinct groups, (ii) corrects the non-normal distribution of a dataset if needed, and (iii) merges two statistically distinct groups in case their effect size - measured using Cliff's Delta (or $d$ ) [39] - is negligible, so that the creation of trivial groups is avoided. To perform the test, we relied on the implementation provided by Tantithamthavorn et al. [114.

\section{4 $\mathbf{R Q}_{2}$ - Comparison between Intensity Index and Antipattern Metrics}

In $\mathbf{R Q}_{2}$ our goal was to compare the performance of change prediction models relying on the intensity index against the one achieved by models exploiting other existing smell-related metrics. In particular, the comparison was done considering the so-called antipattern metrics, which were defined by Taba et al. 110]: these are three metrics aimed at capturing different aspects related to the maintainability of classes affected by code smells. More specifically:

- the Average Number of Antipatterns (ANA) computes how many code smells there were in the previous releases of a class over the total number of releases. This metric is based on the assumption that classes that have been more prone to be smelly in the past are somehow more prone to be smelly in the future;

- the Antipattern Complexity Metric (ACM) computes the entropy of changes involving smelly classes. Such entropy refers to the one originally defined by Hassan [45] in the context of defect prediction. The conjecture behind its use relates to the fact that a more complex development process might lead to the introduction of code smells; 
- the Antipattern Recurrence Length (ARL) measures the total number of subsequent releases in which a class has been affected by a smell. This metric relies on the same underlying conjecture as ANA, i.e., the more a class has been smelly in the past the more it will be smelly in the future.

To compute these metrics, we employed the tool developed by Palomba et al. 85. Then, as done in the context of $\mathbf{R} \mathbf{Q}_{1}$, we plugged the antipattern metrics into the experimented baselines and assess the performance of the resulting change prediction models using the same set of evaluation metrics described in Section 3.3.7, i.e., F-Measure and AUC-ROC. Finally, we statistically compared such performance with the one obtained by the models including the intensity index as predictor.

Besides the comparison in terms of evaluation metrics, we also analyzed the extent to which the two types of models are complementary with respect to the classification of change-prone classes. This was done with the aim of assessing whether the two models, relying on different smell-related information, can correctly identify the change-proneness of different classes. More formally, let $m_{\text {int }}$ be the model built plugging in the intensity index; let $m_{\text {ant }}$ be the model built by considering the antipattern metrics, we computed the following overlap metrics on the set of smelly and change-prone instances of each system:

$$
\begin{aligned}
T P_{m_{i n t} \cap m_{a n t}} & =\frac{\left|T P_{m_{i n t}} \cap T P_{m_{a n t}}\right|}{\left|T P_{m_{i n t}} \cup T P_{m_{a n t}}\right|} \% \\
T P_{m_{i n t} \backslash m_{a n t}} & =\frac{\left|T P_{m_{i n t}} \backslash T P_{m_{a n t}}\right|}{\left|T P_{m_{i n t}} \cup T P_{m_{a n t}}\right|} \% \\
T P_{m_{a n t} \backslash m_{i n t}} & =\frac{\left|T P_{m_{a n t}} \backslash T P_{m_{i n t}}\right|}{\left|T P_{m_{a n t}} \cup T P_{m_{i n t}}\right|} \%
\end{aligned}
$$

where $T P_{m_{\text {int }}}$ represents the set of change-prone classes correctly classified by the prediction model $m_{\text {int }}$, while $T P_{m_{a n t}}$ is the set of change-prone classes correctly classified by the prediction model $m_{\text {ant }}$. The $T P_{m_{\text {int }} \cap m_{\text {ant }}}$ metric measures the overlap between the sets of true positives correctly identified by both models $m_{\text {int }}$ and $m_{\text {ant }}, T P_{m_{\text {int }} \backslash m_{\text {ant }}}$ measures the percentage of change-prone classes correctly classified by $m_{i n t}$ only and missed by $m_{\text {ant }}$, and $T P_{m_{a n t} \backslash m_{\text {int }}}$ measures the percentage of change-prone classes correctly classified by $m_{\text {ant }}$ only and missed by $m_{\text {int }}$.

3.5 $\mathbf{R Q}_{3}$ - Gain Provided by the Intensity Index

In $\mathbf{R Q}_{3}$ we conducted a fine-grained investigation aimed at measuring how important is the intensity index with respect to the other features (i.e., product, process, developer-related, and antipattern metrics) composing the experimented models. To this aim, we used an information gain algorithm 92 to 
quantify the gain provided by adding the intensity index in each prediction model. In our context, this algorithm ranked the features of the models according to their ability to predict the change-proneness of classes. More specifically, let $M$ be a change prediction model, let $P=\left\{p_{1}, \ldots, p_{n}\right\}$ be the set of predictors composing $M$, an information gain algorithm [92] applies the following formula to compute a measure which defines the difference in entropy from before to after the set $M$ is split on an attribute $p_{i}$ :

$$
\operatorname{InfoGain}\left(M, p_{i}\right)=H(M)-H\left(M \mid p_{i}\right)
$$

where the function $H(M)$ indicates the entropy of the model that includes the predictor $p_{i}$, while the function $H\left(M \mid p_{i}\right)$ measures the entropy of the model that does not include $p_{i}$. Entropy is computed as follow:

$$
H(M)=-\sum_{i=1}^{n} \operatorname{prob}\left(p_{i}\right) \log _{2} \operatorname{prob}\left(p_{i}\right)
$$

From a more practical perspective, the algorithm quantifies how much uncertainty in $M$ was reduced after splitting $M$ on predictor $p_{i}$. In our work, we employed the Gain Ratio Feature Evaluation algorithm 92 implemented in the WEKA toolkit [41, which ranks $p_{1}, \ldots, p_{n}$ in descending order based on the contribution provided by $p_{i}$ to the decisions made by $M$. In particular, the output of the algorithm is a ranked list in which the predictors having the higher expected reduction in entropy are placed on the top. Using this procedure, we evaluated the relevance of the predictors in the change prediction models experimented, possibly understanding whether the addition of the intensity index gives a higher contribution with respect to the structural metrics from which it is derived (i.e., metrics used for the detection of the smells) or with respect the other metrics contained in the models.

\section{6 $\mathbf{R Q}_{4}$ - Combining All Predictors and Smell-Related Information}

As a final step of our study, we aimed to study the possibility to devise a combined model able to mix together standard change-proneness predictors (i.e., structural, process, and developer-related metrics) and smell-related information to achieve better prediction performance. To do it, we firstly put all the independent variables considered in the study in a single dataset, thus putting them all together. In the second place, we applied the variable removal procedure based on the vif function (see Section 3.3.2 for details on this technique): in this way, we were able to remove the independent variables that do not significantly influence the performance of the combined model. Finally, we tested the ability of the newly devised model using the same procedures and metrics used in the context of $\mathbf{R Q}_{1}$, i.e., F-measure, AUC-ROC, and Brier score, and statistically comparing the performance of the experimented models by means of Scott-Knott ESD test. 


\section{Analysis of the Results}

F-MEASURE
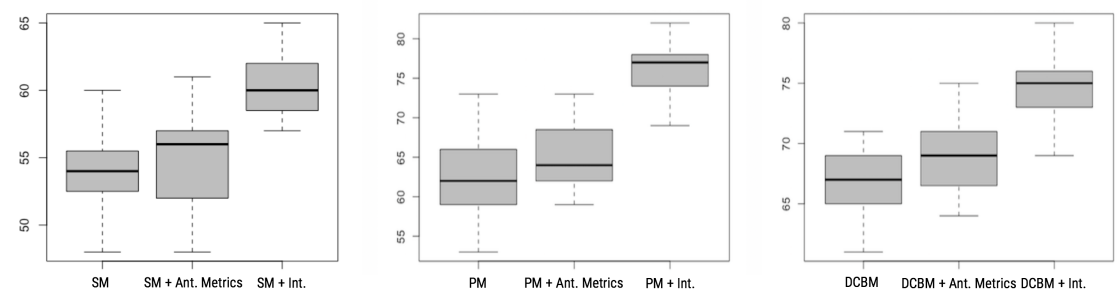

Fig. 1: Overview of the value of F-measure among the models
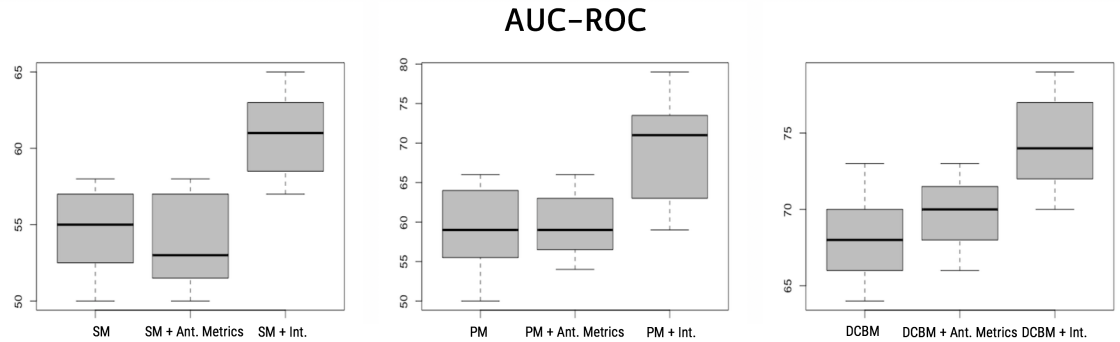

Fig. 2: Overview of the value of AUC-ROC among the models

In this section we report and sum up the results of the presented research questions, discussing the main findings of our study.

4.1 $\mathrm{RQ}_{1}-\mathrm{RQ}_{2}$ : The performance of the intensity-including models and their comparison with the state of the art

Before describing the results related to the contribution of the intensity index in the three prediction models considered, we report the results of the feature selection process aimed at avoiding multi-collinearity. According to the results achieved using the vif function [74, we removed FCH, LCH, WFR, ATAF, CHD, LCD, CSBS, and ACDF from the process-based model [27], while we did not remove any variables from the other baselines. 
Figures 1 and 2 show the box plots reporting the distributions of F-Measure and AUC-ROC achieved by the (i) basic models that do not include any smellrelated information - SM, PM, and DCBM, respectively; (ii) models including the antipattern metrics - those having "+ Ant. Metrics" as suffix; and (iii) models including the intensity index - those reporting "+ Int." as suffix. Note that for the sake of readability, we only report the distribution of F-Measure rather than the distributions of precision and recall. Detailed results for those metrics are available in our online appendix 19.

In the first place, looking at Figure 1, we can observe that the basic model based on scattering metrics (i.e., DCBM) tends to perform better than models built using structural and process metrics. Indeed, DCBM 24 has a median F-Measure $5 \%$ and $13 \%$ higher than structural (67\% vs $54 \%$ ) and process $(67 \%$ vs $62 \%$ ) models, respectively. On the one hand, this result confirms our previous findings on the power of the developer-related factors in change prediction 20]; on the other hand, we can confirm the results achieved by Di Nucci et al. 24 on the value of the scattering metrics for the prediction of problematic classes. As for the role of the intensity index, we notice that with the respect to the SM, PM and DCBM model, the intensity of code smells provides an additional useful information able to increase the ability of the model in discovering change-prone code components. This is observable by looking at the performance in Figures 1 and 2, In the following, we further discuss our findings by reporting our results for each prediction model experimented, comparing the intensity-including ones with the state of the art.

Contribution in Structural-based Models. The addition of the intensity index within the SM model allows the model to reach a median F-Measure of $60 \%$ and an AUC-ROC of $61 \%$, respectively. When compared against the antipattern metrics-including model, the intensity-including one still performs better (i.e., $+4 \%$ in terms of median F-Measure and $+7 \%$ in terms of median AUC-ROC).

Looking deeper into the results, we observed that the shapes of the box plots for the intensity-including model appear less dispersed than the basic one, meaning that the addition of the intensity index makes the performance of the model better and more stable. For instance, considering the Apache-ant-1.3 project, the basic structural model reached $50 \%$ precision and $56 \%$ recall (FMeasure $=53 \%$, while the model that includes the intensity index has a precision of $61 \%$ and a recall of $66 \%(\mathrm{~F}-\mathrm{Measure}=63 \%)$, thus obtaining an improvement of $10 \%$. The same happens all the considered systems: we can therefore claim that the performances of change prediction models strongly improve when considering the intensity of code smells as additional independent variable.

When considering the structural model that includes the antipattern metrics defined by Taba et al. [110, we notice that its performance is just slightly better than the basic model in terms of F-Measure ( $56 \%$ vs $54 \%$ ); more interesting, the AUC-ROC of the "SM + Ant. Metrics" model is lower than the basic one (53\% vs 55\%). From a practical perspective, these results tell us 
that the inclusion of the antipattern metrics only provides slight improvement with respect to the number of actual change-prone classes identified, but at the same time cannot provide benefits in the robustness of the classifications.

In the comparison between the $S M+$ Ant. Metrics and the $S M+$ Int. models, we observe that the performance of the former is always lower than the one achieved by the latter (considering the median of the distributions, $-4 \%$ of F-Measure and - $8 \%$ of AUC-ROC). This indicates that the intensity index can provide much higher benefits in change prediction than existing metrics that capture other smell-related information. Nevertheless, in some cases the antipattern metrics defined by Taba et al. 110 can give complementary information with respect to the intensity index, opening the possibility to obtain still better performance by considering both metric types. Our claim is supported by the overlap analysis shown in Table 7 and computed on the set of change-prone and smelly classes correctly classified by the two models. While $43 \%$ of the instances are correctly classified by both the models, a consistent portion of instances are classified only by SM + Int. model $(35 \%)$ or by the model using the antipattern metrics $(22 \%)$. Consequently, this means that the smell-related information taken into account by the $S M+$ Int. and $S M+A n t$. Metrics models are orthogonal and complement each other.

The observations made above were also confirmed from a statistical point of view. Indeed, the intensity-including prediction model consistently appeared in the top Scott-Knott ESD rank in terms of AUC-ROC, meaning that its performance was statistically higher than the baselines in most of the cases (40 projects out of 43 ).

Contribution in Process-based Models. Also in this case the addition of the intensity index in the model defined by Elish et al. 27 improved its performance with respect to the basic model (PM). Indeed, the overall median value of F-Measure increased of $15 \%$, i.e., F-Measure of $P M+$ Int. is $77 \%$ while that of $P M$ is $62 \%$. An interesting aspect to discuss in this case is related to the ability of the intensity-including model to increase both precision and recall with respect to the basic model. This is, for instance, the case of Apache Ivy 2, where PM reaches $61 \%$ of precision and $49 \%$ of recall; by adding the intensity index, the prediction model increases its performances to $76 \%(+15 \%)$ in terms of precision and $77 \%(+28 \%)$ of recall, demonstrating that a better characterization of the classes having design problems can help in obtaining more accurate predictions.

Looking at the baseline model that includes the antipattern metrics, we notice that it provides improvements when compared to the basic one. However, such improvements are still minor in terms of F-Measure (64\% vs $62 \%)$ and thus we can confirm that the addition of the metrics proposed by Taba et al. [110] does not provide a relevant boost in the performance of basic change prediction models. Similarly, the model based on such metrics is never able to outperform the performance of the intensity-including one, being up to $13 \%$ less performing. At the same time, it is worth reporting an interesting complementarity between the set of change-prone and smelly classes correctly 
classified by "PM + Int." and by the Basic + Ant. Metrics (see Table 7), i.e., the two models correctly capture the change-proneness of different code elements.

The statistical analyses confirmed the findings discussed above. Indeed, the likelihood to be ranked at the top by the Scott-Knott ESD test is always higher for the model including the intensity index. At the same time, the antipattern metrics-including model was confirmed to provide a slight statistical benefits than the basic one (they are ranked in the same cluster in $88 \%$ of the cases).

Contribution in Developer-Related Model. Finally, the results for this type of model is similar to the one discussed above. Indeed, the addition of the intensity in DCBM 24] allows the model to reach a median F-Measure of $75 \%$ and an AUC-ROC of $74 \%$, respectively. When compared to the standard model $D C B M$ the intensity-including one performs better (i.e., $+7 \%$ in terms of median F-Measure and $+6 \%$ in terms of median AUC-ROC). For instance, in the Apache Synapse 1.2 project the "DCBM + Int." obtains an F-Measure and AUC-ROC $12 \%$ and $13 \%$, respectively, higher than $D C B M$. The result holds for all the systems in our dataset, meaning that the addition of the intensity always provides improvements with respect to the baseline.

Comparing the performance of "DCBM + Int." with the model that includes the antipattern metrics [110, we observe that the F-Measure of the former is on average $6 \%$ higher than the latter; the better performance of the intensity-including model is also confirmed when considering the AUC-ROC, which is $4 \%$ higher. Nevertheless, also in this case we found some complementarities in the correct predictions done by these two models (see Table 7): indeed, only $44 \%$ of instances are correctly caught by both the models, while $31 \%$ of them are only captured by "DCBM + Int." and $25 \%$ only by "DCBM + Ant. Metrics". The Scott-Knott ESD test confirmed our observations. The likelihood of the intensity-including model to be ranked at the top is always higher than the other models. At the same time, the antipattern metrics-including models were confirmed to provide statistically better performance than the basic models in $67 \%$ of the considered systems.

Table 7: Overlap analysis between the model including the intensity index and the model including the antipattern metrics.

\begin{tabular}{lccc}
\hline Models & $\begin{array}{c}\text { Int. } \cap \\
\text { Ant.\% }\end{array}$ & $\begin{array}{c}\text { Int. } \\
\text { Ant.\% }\end{array}$ & $\begin{array}{c}\text { Ant. } \\
\text { Int.\% }\end{array}$ \\
\hline SM [128] & 43 & 35 & 22 \\
PM [27] & 47 & 38 & 15 \\
DCBM [24] & 44 & 31 & 25
\end{tabular}


$R_{1}$ - To what extent does the intensity index improve the performance of existing change prediction models? The addition of the intensity index 85 as a predictor of change-prone components increases the performance of the baseline change prediction models in terms of $\mathrm{F}$ Measure up to $10 \%$.

$\mathrm{RQ}_{2}$ - How does the model including the intensity index as predictor compare to a model built using antipattern metrics? The prediction models that include the antipattern metrics [110] only perform slightly better than the basic models, while they have lower performance than the intensity-including ones. However, we observed interesting complementarities between the set of change-prone and smelly classes correctly classified by the models that include intensity index and the models with antipattern metrics, which highlight the possibility to achieve higher performance through a combination of smell-related information.

4.2 $\mathrm{RQ}_{3}$ : Analyzing the gain provided by the intensity index with respect to other predictors

In this section we analyze the results of Gain Ratio Feature Evaluation algorithm [92] in order to understand how important the predictors composing the different models considered in this study are, with the aim to evaluate the predictive power of the intensity index when compared to the other predictors.

Table 8 shows the gain provided by the different predictions employed in the structural metrics-based change prediction model, while Table 9 reports the results for the process-based model and Table 10 those for the DCBM model. In particular, the tables report the ranking of the predictors based on their importance within the individual models through the values of the mean and the standard deviation (computed by considering the results obtained on the single systems) of the expected reduction in entropy caused by partitioning the prediction model according to a given predictor. In addition, we also provide the likelihood of the predictor to be in the top-rank by the Scott-Knott ESD test, i.e., the percentage of times a predictor was statistically better than the others. The following subsections discuss our findings considering each prediction model individually.

Gain Provided to Structural-based Models [128]. The results in Table 8 shows how Coupling Between Objects $(\mathrm{CBO})$ is the metric having the highest predictive power, with an average reduction of entropy of 0.66 and a standard deviation of 0.09 . The Scott-Knott ESD test statistically confirmed the importance of the predictor, since the information gain given by the metric was statistically higher than other metrics in $82 \%$ of the cases. It is worth noting that this result is in line with previous findings in the field [12, 85, 128, which 
Table 8: Gain Provided by Each Metric To The SM Prediction Model.

\begin{tabular}{lccc}
\hline Metric & Mean & St. Dev. & $\begin{array}{c}\text { SK-ESD } \\
\text { Likelihood }\end{array}$ \\
\hline CBO & 0.66 & 0.09 & 82 \\
RFC & 0.61 & 0.05 & 77 \\
Intensity & $\mathbf{0 . 4 9}$ & $\mathbf{0 . 1 3}$ & $\mathbf{7 5}$ \\
LOC & 0.44 & 0.11 & 55 \\
LCOM & 0.43 & 0.12 & 51 \\
Antipattern Complexity Metric & 0.42 & 0.12 & 41 \\
Antipattern Recurrence Length & 0.31 & 0.05 & 32 \\
Average Number of Antipatterns & 0.22 & 0.10 & 21 \\
DIT & 0.13 & 0.02 & 3 \\
\hline
\end{tabular}

showed the relevance of coupling information for the maintainability of software classes. Looking at the ranking, we also noticed that Response For a Class (RFC), Lines of Code (LOC), and Lack of Cohesion of Methods (LCOM3) appear to be relevant. On the one hand, this is still in line with previous findings 12, 85, 128. On the other hand, it is also important to note that our results seem to reconsider the role of code size for assessing change prediction. In particular, unlike the findings by Zhou et al. 128, on the confounding effect of size, we discovered that LOC can be an important predictor to discriminate change-prone classes. This may be due to the large dataset exploited in this study, which allows a higher level of generalizability. The Scott-Knott ESD test confirmed that these metrics are among the most powerful ones.

As for the variable of interest, i.e., the intensity index, we could observe that it is the feature providing the third highest gain in terms of reduction of entropy, as it has a value of Mean and Standard Deviation of 0.49 and 0.13, respectively. Looking at the results of the statistical test, we observed that the intensity index is ranked on the top by the Scott-Knott ESD in $49 \%$ of the cases, thus confirming the high predictive power of the metric. These findings lead to two main observations. In the first place, the intensity index is a relevant variable and for this reason can provide high benefits for the prediction of change-prone classes (as also observed in $\mathbf{R Q}_{1}$ ). Secondly, and perhaps more interesting, the intensity index can be more powerful than other structural metrics from which it is derived: in other words, a metric mixing together different structural aspects to measure how severe a code smell is seems to be more meaningful than the individual metrics used to derive the index.

As for the antipattern metrics, we observed that all of them appear to be less relevant than the intensity index. Somehow, this confirms the results of $\mathbf{R Q}_{2}$, where we showed that adding them to change prediction models results in a limited improvement with respect to the baseline. At the same time, it is worth noting that ACM (i.e., Antipattern Complexity Metric) may sometimes provide a notable contribution. While the average gain is 0.42 , the standard deviation is 0.12 : this means that the entropy reduction can be up to 0.54 , as in the case of the Apache-synapse-2.3. Thus, this result seems to suggest that this metric has some potential for effectively predicting the change-proneness 
of classes. The other two antipattern metrics, i.e., Average Number of Antipatterns (ANA) and Antipattern Recurrence Length (ARL), instead, only provide a partial contribution in the reduction of entropy of the model. Indeed, the mean are 0.31 and 0.22 , respectively, with a standard deviation that is never above 0.1. The Scott-Knott ESD test statistically confirmed the findings: indeed, ACM was a top predictor in $41 \%$ of the datasets, as opposed to ANA and ARL metrics which appeared as statistically more powerful than other metrics only in $32 \%$ and $21 \%$ of the cases. Finally, Depth Inheritance Tree was the less powerful metrics in the ranking, and the Scott-Knott ESD test ranked it at the top in only $3 \%$ of the cases.

Table 9: Gain Provided by Each Metric To The PM Prediction Model.

\begin{tabular}{lccc}
\hline Metric & Mean & St. Dev. & $\begin{array}{c}\text { SK-ESD } \\
\text { Likelihood }\end{array}$ \\
\hline BOC & 0.56 & 0.05 & 75 \\
FRCH & 0.55 & 0.06 & 64 \\
Intensity & $\mathbf{0 . 4 4}$ & $\mathbf{0 . 0 8}$ & $\mathbf{6 1}$ \\
WCD & 0.42 & 0.11 & 55 \\
Antipattern Complexity Metric & 0.41 & 0.04 & 54 \\
LCA & 0.33 & 0.07 & 33 \\
CHO & 0.28 & 0.03 & 31 \\
Antipattern Recurrence Length & 0.24 & 0.05 & 25 \\
Average Number of Antipatterns & 0.09 & 0.03 & 2 \\
CSB & 0.07 & 0.01 & 1 \\
TACH & 0.02 & 0.01 & 1 \\
\hline
\end{tabular}

Gain Provided to Process-based Models [27]. Regarding the process metric-based model considered in this study, the results are similar to the structural model. Indeed, from Table 9 it is evident that the intensity index represents a good predictor; the value of the mean is 0.44 and it is a top predictors in $61 \%$ of the dataset. It appears to be the third most powerful feature of the model, just behind the Birth of a Class and Frequency of Changes. On the one hand, this ranking is pretty expected, as the top two features are those which fundamentally characterize the notion of process-based change prediction proposed by Elish et al. 27]. On the other hand, our findings report that the intensity index can effectively complement the process metrics present in the model, i.e., a structural-based indicator seems to be orthogonal with respect to the other basic features. As for the antipattern metrics, also in this case ACM turned to be a potentially good predictor in $54 \%$ of the dataset, while ANA and ARL are top predictors only in $25 \%$ and $2 \%$ of the dataset, respectively. At the bottom of the ranking there are other basic metrics like Changes since the Birth and Total Amount of Changes: this confirms previous findings 20] reporting that the overall number of previous changes cannot properly model the change-proneness of classes.

Gain Provided to Developer-related factors [24]. Looking at the ranking of the features of the DCBM model, we can still confirm the results discussed so far. Indeed, the intensity index is the second most relevant factor, just 
Table 10: Gain Provided by Each Metric To The DCBM Prediction Model.

\begin{tabular}{lccc}
\hline Metric & Mean & St. Dev. & $\begin{array}{c}\text { SK-ESD } \\
\text { Likelihood }\end{array}$ \\
\hline Semantic Scattering & 0.76 & 0.07 & 95 \\
Intensity & $\mathbf{0 . 7 4}$ & $\mathbf{0 . 0 5}$ & $\mathbf{9 4}$ \\
Structural Scattering & 0.72 & 0.05 & 91 \\
Antipattern Complexity Metric & 0.66 & 0.04 & 78 \\
Antipattern Recurrence Length & 0.31 & 0.02 & 44 \\
Average Number of Antipatterns & 0.11 & 0.03 & 21 \\
\hline
\end{tabular}

behind the semantic scattering: its mean is 0.74, and the Scott-Knott ESD test indicated the intensity index as top predictor in $94 \%$ of the dataset. It is interesting to note that the index provides a higher contribution than the structural scattering, meaning that the combination from which it is derived can provide a higher entropy reduction with respect to a structural metric that computes how far the classes touched by developers in a certain time window are. Regarding the antipattern metrics, the results are similar to those of the other models considered; the ACM provided a pretty high quantity of additional information to the model $($ mean $=0.66)$, being ranked at top predictors in $78 \%$ of the dataset. Instead, the means for ARL and ANA are notably lower (0.31 and 0.11 , respectively), appearing as the least important features.

As a more general observation, it is worth noting that the values of mean information gain of both the intensity index and ACM are much higher $(\approx 0.20$ more) for this model when compared to the structural- and process metricsbased models. This indicates that those metrics can provide a much higher information than the other models: this can be due to the limited number of features employed by this model, which makes the additional metrics more useful to predict the change-proneness of classes.

All in all, we can confirm again that the intensity index provides a strong information gain to all the change prediction models considered in the study, together with ACM from the group of antipattern metrics. This possibly highlights how their combination could provide further improvements in the context of change prediction.

$\mathrm{RQ}_{3}-$ What is the gain provided by the intensity index to change prediction models when compared to other predictors? The intensity index provides a notable information gain in all the considered prediction models. At the same time, a metric of complexity of the change process involving code smells seems to provide further additional information, highlighting the possibility to obtain even better change prediction performance when mixing different smell-related information. 
4.3 $\mathrm{RQ}_{4}$ : The performance of the combined smell-aware bug prediction model

The results achieved in the previous research questions highlight the possibility to build a combined change prediction model that takes into account smellrelated information besides the structural, process, and developer-related metrics. For this reason, in the context of $\mathbf{R Q}_{4}$, we assessed the feasibility of a combined solution and evaluated its performance with respect to the results achieved by the stand-alone models that only rely on structural, process, or developer-related features. As explained in Section 3. to come up with the combined model we firstly put together all the features of the considered models and then applied a feature selection algorithm to discard irrelevant features. Starting from an initial set of 18 metrics, this procedure discarded DIT, CSB, TACH, and ANA. Thus, the combined model comprises 14 metrics: besides most of the basic structural, process, and developer-related predictors, the model includes three smell-related metrics such as (i) intensity index, (ii) $\mathrm{ACM}$, and (iii) ARL.
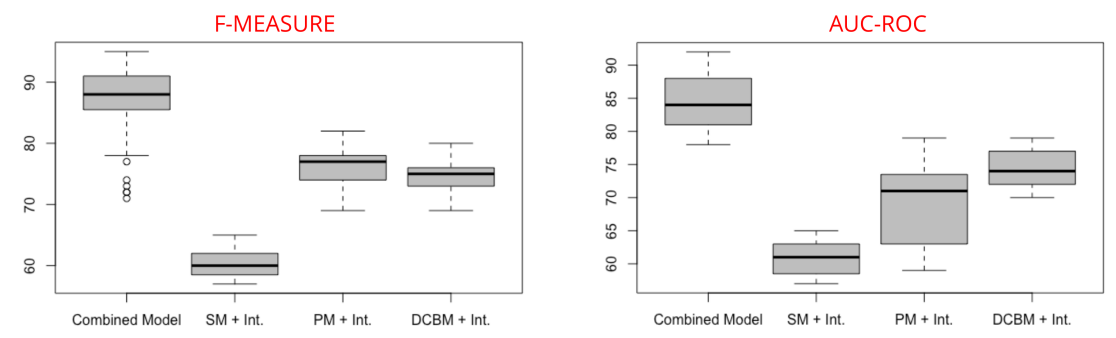

Fig. 3: Overview of the value of F-Measure and AUC-ROC of the Combined Model

Figure 3 shows the boxplots reporting the distributions of F-Measure and AUC-ROC related to the smell-aware combined change prediction model. To facilitate the comparison with the models exploited in the context of $\mathbf{R Q}_{1}$ and $\mathbf{R Q}_{2}$, we also report boxplots depicting the best models coming from our previous analyses, i.e., $S M+$ Int., $P M+$ Int., and $D C B M+$ Int.

Looking at the figures, it seems clear that the combined model has better performances than all the baseline approaches that exploit individual features. Indeed, the median F-Measure reaches $88 \%$, meaning that it is $18 \%$, $11 \%$, and $13 \%$ more accurate than $S M+$ Int., $P M+$ Int., and $D C B M+$ Int., respectively. These results hold when considering the AUC-ROC, where the combined model was able to boost the performance at least $10 \%$ with respect to the basic models that include the intensity. As an example, in the Apache Xalan 2.5 project the best stand-alone model (the "SM+Int" in this case) had an F-Measure close to $73 \%$, while the mixture of features provided by the combined model allowed to reach an F-Measure of 93\%. As expected, the re- 
sults are statistically significant, as the devised smell-aware change prediction model appeared in top Scott-Knott ESD rank in $98 \%$ of the cases.

On the one hand, these strong results confirm previous findings on the importance to combine different predictors of source code maintainability [20, 23]. On the other hand, we can claim that smell-related information [85, 110 improves the capabilities of change prediction models, allowing them to perform much better than other existing models.

$\mathrm{RQ}_{4}$ - What is the performance of a combined change prediction model that includes smell-related information? The devised smell-aware change prediction model performs better than all the baseline approaches considered in the paper, with an F-Measure up to $20 \%$.

\section{Threats to Validity}

In this section we discuss possible threats affecting our results and how we mitigated them.

\subsection{Threats to Construct Validity}

As for threats related to the relationship between theory and observation, they are mainly related to the independent variables used and the dataset exploited. As for the former, we selected state of the art change prediction models based on a different set of basic features, i.e., structural, process, and developer-related metrics, that capture different characteristics of source code. The selection was mainly driven by recent results 20, that showed that the considered models are (i) accurate in the detection of the change-proneness of classes and (ii) complementary to each other, thus correctly identifying different sets of change-prone classes. All in all, this selection process allowed us to test the contribution of smell-related information in different contexts. As for the dataset, we relied on a publicly available source previously built 85. Of course, we cannot exclude possible imprecisions in the computation of the dependent variable. Still in this category, we adopted JCodeOdor 32 to identify code smells and assign to them a level of intensity. Our choice was driven by previous results 85 that showed, on the same dataset, that this tool has a high accuracy (i.e., F-Measure $=80 \%$ ). Despite this performance, the tool still identifies 154 false positives and 94 false negatives among the 43 considered systems. To make the set of code smells as close as possible to the ground truth, in our study we manually elaborated on the output of the tool by (i) setting to zero the intensity index of the false positive instances, and (ii) discarding the false negatives, i.e., the instances for which we could not assign an intensity value. Since this manual process is not always feasible, we also evaluated the effect of including false positive and false negative instances in 
the construction of the change prediction models. More specifically, we re-ran the analyses performed in Section 3 and validated the performance of the experimented models when including the false positive instances using the same metrics used to assess the performance of the other prediction models (i.e., FMeasure and AUC-ROC). Our results report that these models always perform better than other models that do not include any smell-related information, while they are slightly less performing (-3\% in terms of median F-Measure) than those built discarding the false positive instances.

In the second place, we evaluated what is the impact of including false negative instances. Their intensity index is, by definition, equal to zero: as a consequence, they were considered in the same way as non-smelly classes. The results of our analyses showed that the intensity-including models still produce better results than those of the baselines, as they boosted their median FMeasure of $\approx 4 \%$. At the same time, we observed a decrement of $2 \%$ in terms of F-Measure with respect to the performance obtained by the prediction models built discarding false negatives.

As a final step, we also considered the case where both false positives and false negatives are incorporated in the experimented models. Our findings report that the Basic + Intensity models have a median F-Measure $2 \%$ lower than the models where the false positive and false negative instances were filtered out. At the same time, they were still better than basic models (median F-Measure $=+6 \%$ ). Thus, we could conclude that a fully automatic code smell detection process still provides better performance than existing change prediction models. This result is, in our opinion, extremely valuable as it indicates that practitioners can adopt automatic code smell detectors without the need of manually investigating the candidates they give as output.

It is worth remarking that the choice of considering code smell severity rather than the simple presence/absence of smells was driven by the conjecture that the severity can give a more fine-grained information on how much a design problem is harmful for a certain source code class. To verify this conjecture, we conducted a further analysis aimed at establishing the performance of the experimented models where considering a boolean value reporting the presence of code smells rather than their intensity. As expected, our findings reported that the models relying on the intensity were more powerful than those based on the boolean indication of the smell presence. This further confirms the idea behind this paper, i.e., code smell intensity can improve change-proneness prediction.

Our observations might still have been threatened by the presence of a large number of code smell co-occurrences [86, 125], which might have biased the intensity level of the smelly classes of our dataset. To account for this aspect, we measured the percentage of classes in our dataset affected by more than one smell: we only found that $8 \%$ of the classes, on average, contained more code smells. As a consequence, we can claim that the problem of co-occurrence is rather limited in our study.

A further threat to construct validity relates to the dataset exploited in our empirical study. In this regard, we rely on a publicly available oracles from the 
PROMISE repository [67], however we performed some preliminary operations to ensure data quality and robustness, by employing the data preprocessing algorithm implementing the guidelines by Shepperd et al. [103] aimed at removing noisy and/or erroneous data items.

\subsection{Threats to Conclusion Validity}

Threats to conclusion validity refer to the relation between treatment and outcome. When evaluating the change prediction models we computed wellestablished metrics such as F-Measure and AUC-ROC. Furthermore, we statistically verified the differences in the performance achieved by the different experimented models using the Scott-Knott statistical test 98 and Cliff's Delta 40 for measuring the effect size. Moreover, we analyzed to what extent the intensity index is important with respect to the other metrics by analyzing the gain provided by the addition of the severity measure in the model. Finally, to ensure that the experimented models did not suffer from multi-collinearity, we adopted the variance inflation factors function [74 to discard non-relevant variables from the considered features.

Still in this category there is a possible threat related to the validation methodology exploited. As shown by Tantithamthavorn et al. 114, ten-fold cross validation might provide unstable results because of the effect of random splitting. To deal with this issue, we repeated the 10 -fold cross validation 100 times: in this way, we drastically removed the bias due to the validation strategy.

\subsection{Threats to External Validity}

Threats in this category mainly concern the generalization of results. In this case we analyzed a large set of 43 releases of 14 software systems coming from different application domains and with different characteristics (size, number of classes, etc.). Another threat in this category regards the choice of the baseline models. However, we evaluated the contribution of the smell-related information in the context of change prediction models widely used in the past [20, 27, 128] that take into account predictors of different nature, i.e., , product, process, and developer-related metrics. However, we are aware that our study is based on systems developed in Java only, and therefore future investigations aimed at corroborating our findings on a different set of systems would be worthwhile.

\section{Conclusion}

Change-prone classes represent code components that tend to change more often, because of their importance for the business logic of a system or because 
not properly designed by developers. To help practitioners in keeping maintenance and evolution activities under control, many change prediction models have been defined in literature [20, 27, 128. Following the findings of previous studies [52, 83] reporting the impact of code smells on the change-proneness of classes, in this paper we aimed at investigating the impact of smell-related information for the prediction of change-prone classes. We first conducted a large empirical study on 43 releases of 14 software systems and evaluated the contribution of the intensity index proposed by Arcelli Fontana et al. 4 within existing change prediction models based on structural- [128, process- 27, and developer-related 24] metrics. We also compared the gain provided by the intensity index with the one given by the so-called antipattern metrics [110], i.e., metrics capturing historical aspects of code smells.

The results indicated how the addition of the intensity index as a predictor of change-prone components increases the performance of baseline change prediction models by an average of $10 \%$ in terms of F-Measure $\left(\mathrm{RQ}_{1}\right)$. Moreover, the intensity index can boost the performance of such models more than state of the art smell-related metrics such as those defined by Taba et al. [110, even though we observed some complementarities between the models exploiting different information on code smells $\left(\mathrm{RQ}_{2}\right)$. Based on these results, we built a combined smell-aware change prediction model that takes into account product, process, developer- and smell-related information $\left(\mathrm{RQ}_{4}\right)$. The key results showed how the combined model provides a consistent boost in terms of F-Measure, which goes up to $20 \%$ more.

Our findings represent the main input for our future research agenda: we first aim at further testing the usefulness of the devised model in an industrial setting. Furthermore, we plan to perform a fine-grained analysis into the role of each smell type independently on the change prediction power.

\section{References}

1. Abbes M, Khomh F, Gueheneuc YG, Antoniol G (2011) An empirical study of the impact of two antipatterns, blob and spaghetti code, on program comprehension. In: Software maintenance and reengineering (CSMR), 2011 15th European conference on, IEEE, pp 181-190

2. Abdi M, Lounis H, Sahraoui H (2006) Analyzing change impact in objectoriented systems. In: 32nd EUROMICRO Conference on Software Engineering and Advanced Applications (EUROMICRO'06), IEEE, pp 310319

3. Aniche M, Treude C, Zaidman A, van Deursen A, Gerosa M (2016) Satt: Tailoring code metric thresholds for different software architectures. In: 2016 IEEE 16th IEEE International Working Conference on Source Code Analysis and Manipulation (SCAM)

4. Arcelli Fontana F, Ferme V, Zanoni M, Roveda R (2015) Towards a prioritization of code debt: A code smell intensity index. In: Proceedings of the Seventh International Workshop on Managing Technical Debt (MTD 
2015), IEEE, Bremen, Germany, pp 16-24, in conjunction with ICSME 2015

5. Arcelli Fontana F, Mäntylä MV, Zanoni M, Marino A (2016) Comparing and experimenting machine learning techniques for code smell detection. Empirical Software Engineering 21(3):1143-1191, DOI 10.1007/s10664-015-9378-4, URL http://dx.doi.org/10.1007/ s10664-015-9378-4

6. Arisholm E, Briand LC, Foyen A (2004) Dynamic coupling measurement for object-oriented software. IEEE Transactions on Software Engineering 30(8):491-506

7. Bacchelli A, Bird C (2013) Expectations, outcomes, and challenges of modern code review. In: Proc. of the International Conference on Software Engineering (ICSE), IEEE, pp 712-721

8. Baeza-Yates R, Ribeiro-Neto B (1999) Modern Information Retrieval. Addison-Wesley

9. Baeza-Yates R, Ribeiro-Neto B, et al (1999) Modern information retrieval, vol 463. ACM press New York

10. Baeza-Yates RA, Ribeiro-Neto B (1999) Modern Information Retrieval. Addison-Wesley Longman Publishing Co., Inc.

11. Bansiya J, Davis CG (2002) A hierarchical model for object-oriented design quality assessment. IEEE Trans Softw Eng 28(1):4-17

12. Basili VR, Briand LC, Melo WL (1996) A validation of object-oriented design metrics as quality indicators. IEEE Trans Softw Eng 22(10):751761

13. Bell RM, Ostrand TJ, Weyuker EJ (2013) The limited impact of individual developer data on software defect prediction. Empirical Softw Engg 18(3):478-505

14. Bieman JM, Straw G, Wang H, Munger PW, Alexander RT (2003) Design patterns and change proneness: an examination of five evolving systems. In: Proc. Int'l Workshop on Enterprise Networking and Computing in Healthcare Industry, pp 40-49, DOI 10.1109/METRIC.2003.1232454

15. Bottou L, Vapnik V (1992) Local learning algorithms. Neural Comput 4(6):888-900

16. Boussaa M, Kessentini W, Kessentini M, Bechikh S, Ben Chikha S (2013) Competitive coevolutionary code-smells detection. In: Search Based Software Engineering, Lecture Notes in Computer Science, vol 8084, Springer Berlin Heidelberg, pp 50-65

17. Briand LC, Wust J, Lounis H (1999) Using coupling measurement for impact analysis in object-oriented systems. In: Proc. Int'l Conf. on Software Maintenance (ICSM), IEEE, pp 475-482

18. Catolino G, Ferrucci F (2018) Ensemble techniques for software change prediction: A preliminary investigation. In: Machine Learning Techniques for Software Quality Evaluation (MaLTeSQuE), 2018 IEEE Workshop on, IEEE, pp 25-30

19. Catolino G, Palomba F, Arcelli Fontana F, De Lucia A, Ferrucci F, Zaidman A (2018) Improving change prediction models with code smell- 
related information - replication package - https://figshare.com/s/ f536bb37f3790914a32a

20. Catolino G, Palomba F, De Lucia A, Ferrucci F, Zaidman A (2018) Enhancing change prediction models using developer-related factors. Journal of Systems and Software

21. le Cessie S, van Houwelingen J (1992) Ridge estimators in logistic regression. Applied Statistics 41(1):191-201

22. D'Ambros M, Bacchelli A, Lanza M (2010) On the impact of design flaws on software defects. In: Quality Software (QSIC), 2010 10th International Conference on, pp 23-31, DOI 10.1109/QSIC.2010.58

23. DAmbros M, Lanza M, Robbes R (2012) Evaluating defect prediction approaches: a benchmark and an extensive comparison. Empirical Software Engineering 17(4):531-577

24. Di Nucci D, Palomba F, De Rosa G, Bavota G, Oliveto R, De Lucia A (2017) A developer centered bug prediction model. IEEE Trans on Softw Engineering $\mathrm{p}$ to appear

25. Di Nucci D, Palomba F, Tamburri DA, Serebrenik A, De Lucia A (2018) Detecting code smells using machine learning techniques: are we there yet? In: 2018 IEEE 25th International Conference on Software Analysis, Evolution and Reengineering (SANER), IEEE, pp 612-621

26. Di Penta M, Cerulo L, Gueheneuc YG, Antoniol G (2008) An empirical study of the relationships between design pattern roles and class change proneness. In: Proc. Int'l Conf. on Software Maintenance (ICSM), IEEE, pp 217-226, DOI 10.1109/ICSM.2008.4658070

27. Elish MO, Al-Rahman Al-Khiaty M (2013) A suite of metrics for quantifying historical changes to predict future change-prone classes in objectoriented software. Journal of Software: Evolution and Process 25(5):407437

28. Eski S, Buzluca F (2011) An empirical study on object-oriented metrics and software evolution in order to reduce testing costs by predicting change-prone classes. In: Proc. Int'l Conf Software Testing, Verification and Validation Workshops (ICSTW), IEEE, pp 566-571

29. Fluri B, Wuersch M, PInzger M, Gall H (2007) Change distilling: Tree differencing for fine-grained source code change extraction. IEEE Transactions on software engineering 33(11)

30. Fontana FA, Zanoni M (2017) Code smell severity classification using machine learning techniques. Knowledge-Based Systems 128:43-58

31. Fontana FA, Zanoni M, Marino A, Mantyla MV (2013) Code smell detection: Towards a machine learning-based approach. In: Software Maintenance (ICSM), 2013 29th IEEE International Conference on, pp 396-399, DOI 10.1109/ICSM.2013.56

32. Fontana FA, Ferme V, Zanoni M, Roveda R (2015) Towards a prioritization of code debt: A code smell intensity index. In: 2015 IEEE 7th International Workshop on Managing Technical Debt (MTD), IEEE, pp $16-24$ 
33. Fontana FA, Ferme V, Zanoni M, Yamashita A (2015) Automatic metric thresholds derivation for code smell detection. In: Proceedings of the Sixth international workshop on emerging trends in software metrics, IEEE Press, pp 44-53

34. Fontana FA, Dietrich J, Walter B, Yamashita A, Zanoni M (2016) Antipattern and code smell false positives: Preliminary conceptualization and classification. In: 2016 IEEE 23rd International Conference on Software Analysis, Evolution, and Reengineering (SANER), vol 1, pp 609613, DOI 10.1109/SANER.2016.84

35. Fowler M, Beck K, Brant J, Opdyke W, Roberts D (1999) Refactoring: Improving the Design of Existing Code. Addison-Wesley

36. Gatrell M, Counsell S (2015) The effect of refactoring on change and fault-proneness in commercial c\# software. Science of Computer Programming 102(0):44 - 56, DOI http://dx.doi.org/10.1016/j.scico.2014. 12.002, URL http://www.sciencedirect.com/science/article/pii/ S0167642314005711

37. Ghotra B, McIntosh S, Hassan AE (2015) Revisiting the impact of classification techniques on the performance of defect prediction models. In: Proceedings of the International Conference on Software Engineering, IEEE, pp 789-800

38. Girba T, Ducasse S, Lanza M (2004) Yesterday's weather: Guiding early reverse engineering efforts by summarizing the evolution of changes. In: Proc. Int'l Conf. Softw. Maintenance (ICSM), IEEE, pp 40-49

39. Grissom RJ, Kim JJ (2005) Effect sizes for research: A broad practical approach, 2nd edn. Lawrence Earlbaum Associates

40. Grissom RJ, Kim JJ (2005) Effect sizes for research: A broad practical approach, 2nd edn. Lawrence Earlbaum Associates

41. Hall M, Frank E, Holmes G, Pfahringer B, Reutemann P, Witten IH (2009) The weka data mining software: An update. SIGKDD Explor Newsl 11(1):10-18, DOI 10.1145/1656274.1656278, URL http://doi. acm.org/10.1145/1656274.1656278

42. Hall T, Beecham S, Bowes D, Gray D, Counsell S (2011) Developing faultprediction models: What the research can show industry. IEEE Software 28(6):96-99

43. Han AR, Jeon SU, Bae DH, Hong JE (2008) Behavioral dependency measurement for change-proneness prediction in uml 2.0 design models. In: 32nd Annual IEEE International Computer Software and Applications Conference, IEEE, pp 76-83

44. Han AR, Jeon SU, Bae DH, Hong JE (2010) Measuring behavioral dependency for improving change-proneness prediction in uml-based design models. Journal of Systems and Software 83(2):222-234

45. Hassan AE (2009) Predicting faults using the complexity of code changes. In: Int'l Conf. Software Engineering (ICSE), IEEE, pp 78-88

46. John GH, Langley P (1995) Estimating continuous distributions in bayesian classifiers. In: Eleventh Conference on Uncertainty in Artificial Intelligence, Morgan Kaufmann, San Mateo, pp 338-345 
47. Kennedy J (2011) Particle swarm optimization. In: Encyclopedia of machine learning, Springer, pp 760-766

48. Kessentini M, Vaucher S, Sahraoui H (2010) Deviance from perfection is a better criterion than closeness to evil when identifying risky code. In: Proceedings of the IEEE/ACM International Conference on Automated Software Engineering, ACM, ASE '10, pp 113-122

49. Kessentini W, Kessentini M, Sahraoui H, Bechikh S, Ouni A (2014) A cooperative parallel search-based software engineering approach for codesmells detection. IEEE Transactions on Software Engineering 40(9):841861, DOI 10.1109/TSE.2014.2331057

50. Khomh F, Di Penta M, Gueheneuc YG (2009) An exploratory study of the impact of code smells on software change-proneness. In: 2009 16th Working Conference on Reverse Engineering, IEEE, pp 75-84

51. Khomh F, Vaucher S, Guéhéneuc YG, Sahraoui H (2009) A bayesian approach for the detection of code and design smells. In: Proceedings of the International Conference on Quality Software (QSIC), IEEE, Hong Kong, China, pp 305-314

52. Khomh F, Di Penta M, Guéhéneuc YG, Antoniol G (2012) An exploratory study of the impact of antipatterns on class change-and faultproneness. Empirical Softw Engg 17(3):243-275

53. Kohavi R (1995) The power of decision tables. In: 8th European Conference on Machine Learning, Springer, pp 174-189

54. Kumar L, Behera RK, Rath S, Sureka A (2017) Transfer learning for cross-project change-proneness prediction in object-oriented software systems: A feasibility analysis. ACM SIGSOFT Software Engineering Notes $42(3): 1-11$

55. Kumar L, Rath SK, Sureka A (2017) Empirical analysis on effectiveness of source code metrics for predicting change-proneness. In: ISEC, pp 4-14

56. Lanza M, Marinescu R (2006) Object-Oriented Metrics in Practice: Using Software Metrics to Characterize, Evaluate, and Improve the Design of Object-Oriented Systems. Springer

57. Le Cessie S, Van Houwelingen JC (1992) Ridge estimators in logistic regression. Applied statistics pp 191-201

58. Lehman MM, Belady LA (eds) (1985) Program Evolution: Processes of Software Change. Academic Press Professional, Inc.

59. Lu H, Zhou Y, Xu B, Leung H, Chen L (2012) The ability of objectoriented metrics to predict change-proneness: a meta-analysis. Empirical software engineering 17(3):200-242

60. Malhotra R, Bansal A (2015) Predicting change using software metrics: A review. In: Int'l Conf. on Reliability, Infocom Technologies and Optimization (ICRITO), IEEE, pp 1-6

61. Malhotra R, Khanna M (2013) Investigation of relationship between object-oriented metrics and change proneness. International Journal of Machine Learning and Cybernetics 4(4):273-286

62. Malhotra R, Khanna M (2014) A new metric for predicting software change using gene expression programming. In: Proc. Int'l Workshop on 
Emerging Trends in Software Metrics, ACM, pp 8-14

63. Malhotra R, Khanna M (2017) Software change prediction using voting particle swarm optimization based ensemble classifier. In: Proc. of the Genetic and Evolutionary Computation Conference Companion, ACM, pp 311-312

64. Marinescu C (2014) How good is genetic programming at predicting changes and defects? In: Int'l Symp. on Symbolic and Numeric Algorithms for Scientific Computing (SYNASC), IEEE, pp 544-548

65. Marinescu R (2004) Detection strategies: Metrics-based rules for detecting design flaws. In: Proceedings of the International Conference on Software Maintenance (ICSM), pp 350-359

66. Marinescu R (2012) Assessing technical debt by identifying design flaws in software systems. IBM Journal of Research and Development 56(5):9-1

67. Menzies T, Caglayan B, Kocaguneli E, Krall J, Peters F, Turhan B (2012) The promise repository of empirical software engineering data

68. Miryung Kim NN Tom Zimmermann (2014) An empirical study of refactoring challenges and benefits at Microsoft. IEEE Transactions on Software Engineering 40

69. Mkaouer MW, Kessentini M, Bechikh S, Cinnéide MÓ (2014) A robust multi-objective approach for software refactoring under uncertainty. In: International Symposium on Search Based Software Engineering, Springer, pp 168-183

70. Moha N, Guéhéneuc YG, Duchien L, Meur AFL (2010) Decor: A method for the specification and detection of code and design smells. IEEE Transactions on Software Engineering 36(1):20-36

71. Morales R, Soh Z, Khomh F, Antoniol G, Chicano F (2016) On the use of developers' context for automatic refactoring of software anti-patterns. Journal of Systems and Software (JSS)

72. Munro MJ (2005) Product metrics for automatic identification of "bad smell" design problems in java source-code. In: Proceedings of the International Software Metrics Symposium (METRICS), IEEE, p 15

73. Murphy-Hill E, Black AP (2010) An interactive ambient visualization for code smells. In: Proceedings of the 5th international symposium on Software visualization, ACM, pp 5-14

74. O'brien RM (2007) A caution regarding rules of thumb for variance inflation factors. Quality \& Quantity 41(5):673-690

75. Olbrich SM, Cruzes DS, Sjberg DIK (2010) Are all code smells harmful? a study of god classes and brain classes in the evolution of three open source systems. In: in: Intl Conf. Softw. Maint, pp 1-10

76. Oliveto R, Khomh F, Antoniol G, Guéhéneuc YG (2010) Numerical signatures of antipatterns: An approach based on B-splines. In: Proceedings of the European Conference on Software Maintenance and Reengineering (CSMR), IEEE, pp 248-251

77. Palomba F, Zaidman A (2017) Does refactoring of test smells induce fixing flaky tests? In: Software Maintenance and Evolution (ICSME), 2017 IEEE International Conference on, IEEE, pp 1-12 
78. Palomba F, Bavota G, Di Penta M, Oliveto R, De Lucia A, Poshyvanyk D (2013) Detecting bad smells in source code using change history information. In: Automated software engineering (ASE), 2013 IEEE/ACM 28th international conference on, IEEE, pp 268-278

79. Palomba F, Bavota G, Di Penta M, Oliveto R, De Lucia A (2014) Do they really smell bad? a study on developers' perception of bad code smells. In: Software maintenance and evolution (ICSME), 2014 IEEE international conference on, IEEE, pp 101-110

80. Palomba F, Bavota G, Di Penta M, Oliveto R, Poshyvanyk D, De Lucia A (2015) Mining version histories for detecting code smells. IEEE Transactions on Software Engineering 41(5):462-489, DOI 10.1109/TSE.2014. 2372760

81. Palomba F, Lucia AD, Bavota G, Oliveto R (2015) Anti-pattern detection: Methods, challenges, and open issues. Advances in Computers 95:201-238, DOI 10.1016/B978-0-12-800160-8.00004-8

82. Palomba F, Panichella A, Zaidman A, Oliveto R, De Lucia A (2016) A textual-based technique for smell detection. In: Proceedings of the 24th International Conference on Program Comprehension (ICPC 2016), IEEE, Austin, USA, p to appear

83. Palomba F, Bavota G, Di Penta M, Fasano F, Oliveto R, De Lucia A (2017) On the diffuseness and the impact on maintainability of code smells: a large scale empirical investigation. Empirical Software Engineering pp 1-34

84. Palomba F, Panichella A, Zaidman A, Oliveto R, De Lucia A (2017) The scent of a smell: An extensive comparison between textual and structural smells. Transactions on Software Engineering

85. Palomba F, Zanoni M, Fontana FA, De Lucia A, Oliveto R (2017) Toward a smell-aware bug prediction model. IEEE Transactions on Software Engineering

86. Palomba F, Bavota G, Di Penta M, Fasano F, Oliveto R, De Lucia A (2018) A large-scale empirical study on the lifecycle of code smell cooccurrences. Information and Software Technology 99:1-10

87. Palomba F, Zaidman A, De Lucia A (2018) Automatic Test Smell Detection using Information Retrieval Techniques. In: International Conference on Software Maintenance and Evolution (ICSME), IEEE, p to appear

88. Parnas DL (1994) Software aging. In: Proc. of the International Conference on Software Engineering (ICSE), IEEE, pp 279-287

89. Peer A, Malhotra R (2013) Application of adaptive neuro-fuzzy inference system for predicting software change proneness. In: Advances in Computing, Communications and Informatics (ICACCI), 2013 International Conference on, IEEE, pp 2026-2031

90. Peng CYJ, Lee KL, Ingersoll GM (2002) An introduction to logistic regression analysis and reporting. The Journal of Educational Research 96(1):3-14

91. Peters R, Zaidman A (2012) Evaluating the lifespan of code smells using software repository mining. In: Software Maintenance and Reengineering 
(CSMR), 2012 16th European Conference on, IEEE, pp 411-416

92. Quinlan JR (1986) Induction of decision trees. Mach Learn 1(1):81-106, DOI 10.1023/A:1022643204877, URL http://dx.doi.org/10.1023/A : 1022643204877

93. Ratiu D, Ducasse S, Gîrba T, Marinescu R (2004) Using history information to improve design flaws detection. In: Proceedings of the European Conference on Software Maintenance and Reengineering (CSMR), IEEE, pp 223-232

94. Romano D, Pinzger M (2011) Using source code metrics to predict change-prone java interfaces. In: Proc. Int'l Conf. Software Maintenance (ICSM), IEEE, pp 303-312

95. Rosenblatt F (1961) Principles of Neurodynamics: Perceptrons and the Theory of Brain Mechanisms. Spartan Books

96. Rumbaugh J, Jacobson I, Booch G (2004) Unified Modeling Language Reference Manual, The (2Nd Edition). Pearson Higher Education

97. Sahin D, Kessentini M, Bechikh S, Deb K (2014) Code-smell detection as a bilevel problem. ACM Trans Softw Eng Methodol 24(1):6:1-6:44, DOI $10.1145 / 2675067$

98. Scott AJ, Knott M (1974) A cluster analysis method for grouping means in the analysis of variance. Biometrics 30:507-512

99. Sharafat AR, Tahvildari L (2007) A probabilistic approach to predict changes in object-oriented software systems. In: Proc. Conf. on Softw. Maintenance and Reengineering (CSMR), IEEE, pp 27-38

100. Sharafat AR, Tahvildari L (2008) Change prediction in object-oriented software systems: A probabilistic approach. Journal of Software 3(5):2639

101. Shepperd M, Song Q, Sun Z, Mair C (2013) Data quality: Some comments on the nasa software defect datasets. IEEE Transactions on Software Engineering 39(9):1208-1215

102. Shepperd M, Bowes D, Hall T (2014) Researcher bias: The use of machine learning in software defect prediction. IEEE Transactions on Software Engineering 40(6):603-616, DOI 10.1109/TSE.2014.2322358

103. Shepperd M, Bowes D, Hall T (2014) Researcher bias: The use of machine learning in software defect prediction. IEEE Trans on Softw Engineering 40(6):603-616

104. Sjoberg DI, Yamashita A, Anda BC, Mockus A, Dyba T (2013) Quantifying the effect of code smells on maintenance effort. IEEE Transactions on Software Engineering (8):1144-1156

105. Soetens QD, Demeyer S, Zaidman A, Pérez J (2016) Change-based test selection: An empirical evaluation. Empirical Softw Engg 21(5):19902032

106. Song Q, Jia Z, Shepperd M, Ying S, Liu J (2011) A general software defect-proneness prediction framework. IEEE Transactions on Software Engineering 37(3):356-370, DOI 10.1109/TSE.2010.90

107. Spadini D, Palomba F, Zaidman A, Bruntink M, Bacchelli A (2018) On The Relation of Test Smells to Software Code Quality. In: International 
Conference on Software Maintenance and Evolution (ICSME), IEEE, p to appear

108. Spinellis D (2005) Tool writing: A forgotten art? IEEE Software (4):9-11

109. Stone M (1974) Cross-validatory choice and assessment of statistical predictions. Journal of the royal statistical society Series B (Methodological) pp 111-147

110. Taba SES, Khomh F, Zou Y, Hassan AE, Nagappan M (2013) Predicting bugs using antipatterns. In: Proceedings of the 2013 IEEE International Conference on Software Maintenance, IEEE Computer Society, Washington, DC, USA, ICSM '13, pp 270-279, DOI 10.1109/ICSM.2013.38, URL http://dx.doi.org/10.1109/ICSM.2013.38

111. Taibi D, Janes A, Lenarduzzi V (2017) How developers perceive smells in source code: A replicated study. Information and Software Technology 92:223-235

112. Tantithamthavorn C, McIntosh S, Hassan AE, Matsumoto K (2016) Automated parameter optimization of classification techniques for defect prediction models. In: Proceedings of the 38th International Conference on Software Engineering, ACM, New York, NY, USA, ICSE '16, pp 321-332, DOI 10.1145/2884781.2884857, URL http://doi .acm.org/ $10.1145 / 2884781.2884857$

113. Tantithamthavorn C, McIntosh S, Hassan AE, Matsumoto K (2016) Comments on researcher bias: The use of machine learning in software defect prediction. IEEE Transactions on Software Engineering 42(11):10921094, DOI 10.1109/TSE.2016.2553030

114. Tantithamthavorn C, McIntosh S, Hassan AE, Matsumoto K (2017) An empirical comparison of model validation techniques for defect prediction models. IEEE Trans Softw Eng 43(1):1-18, DOI 10.1109/TSE.2016. 2584050, URL https://doi.org/10.1109/TSE. 2016.2584050

115. Tempero E, Anslow C, Dietrich J, Han T, Li J, Lumpe M, Melton H, Noble J (2010) The qualitas corpus: A curated collection of java code for empirical studies. In: Proc. 17th Asia Pacific Software Eng. Conf., IEEE, Sydney, Australia, pp 336-345, DOI 10.1109/APSEC.2010.46

116. Theodoridis S, Koutroumbas K (2008) Pattern recognition. IEEE Transactions on Neural Networks 19(2):376-376

117. Tsantalis N, Chatzigeorgiou A (2009) Identification of move method refactoring opportunities. IEEE Transactions on Software Engineering $35(3): 347-367$

118. Tsantalis N, Chatzigeorgiou A, Stephanides G (2005) Predicting the probability of change in object-oriented systems. IEEE Transactions on Software Engineering 31(7):601-614

119. Tufano M, Palomba F, Bavota G, Oliveto R, Di Penta M, De Lucia A, Poshyvanyk D (2015) When and why your code starts to smell bad. In: Proceedings of the 37th International Conference on Software Engineering-Volume 1, IEEE Press, pp 403-414

120. Tufano M, Palomba F, Bavota G, Di Penta M, Oliveto R, De Lucia A, Poshyvanyk D (2016) An empirical investigation into the nature of test 
smells. In: Proceedings of the 31st IEEE/ACM International Conference on Automated Software Engineering, pp 4-15

121. Tufano M, Palomba F, Bavota G, Oliveto R, Di Penta M, De Lucia A, Poshyvanyk D (2017) When and why your code starts to smell bad (and whether the smells go away). IEEE Transactions on Software Engineering p to appear.

122. Vidal S, Guimaraes E, Oizumi W, Garcia A, Pace AD, Marcos C (2016) On the criteria for prioritizing code anomalies to identify architectural problems. In: Proceedings of the 31st Annual ACM Symposium on Applied Computing, ACM, pp 1812-1814

123. Vidal SA, Marcos C, Díaz-Pace JA (2016) An approach to prioritize code smells for refactoring. Automated Software Engineering 23(3):501-532

124. Y Freund LM (1999) The alternating decision tree learning algorithm. In: Proceeding of the Sixteenth International Conference on Machine Learning, pp 124-133

125. Yamashita A, Moonen L (2013) Exploring the impact of inter-smell relations on software maintainability: An empirical study. In: Proceedings of the International Conference on Software Engineering (ICSE), IEEE, pp 682-691

126. Yamashita AF, Moonen L (2012) Do code smells reflect important maintainability aspects? In: Proceedings of the International Conference on Software Maintenance (ICSM), IEEE, pp 306-315

127. Zhao L, Hayes JH (2011) Rank-based refactoring decision support: two studies. Innovations in Systems and Software Engineering 7(3):171

128. Zhou Y, Leung H, Xu B (2009) Examining the potentially confounding effect of class size on the associations between object-oriented metrics and change-proneness. IEEE Transactions on Software Engineering 35(5):607-623 